\title{
Validation of an auralization system
}

\author{
Christensen, Claus Lynge; Weitze, Christoffer Andreas; Rindel, Jens Holger; Gade, Anders Christian
}

\section{Published in:}

Acoustical Society of America. Journal

Link to article, DOI:

$10.1121 / 1.1486347$

Publication date:

2001

Document Version

Publisher's PDF, also known as Version of record

Link back to DTU Orbit

Citation (APA):

Christensen, C. L., Weitze, C. A., Rindel, J. H., \& Gade, A. C. (2001). Validation of an auralization system. Acoustical Society of America. Journal, 111(5), 2389-2389. https://doi.org/10.1121/1.1486347

\section{General rights}

Copyright and moral rights for the publications made accessible in the public portal are retained by the authors and/or other copyright owners and it is a condition of accessing publications that users recognise and abide by the legal requirements associated with these rights.

- Users may download and print one copy of any publication from the public portal for the purpose of private study or research.

- You may not further distribute the material or use it for any profit-making activity or commercial gain

- You may freely distribute the URL identifying the publication in the public portal

If you believe that this document breaches copyright please contact us providing details, and we will remove access to the work immediately and investigate your claim 


\title{
Session 3aAAa
}

\section{Architectural Acoustics: Computer Modeling in Acoustical Consulting II}

\author{
Lily M. Wang, Chair \\ Architectural Engineering, University of Nebraska, 200B Peter Kiewit Institute, 1110 South 67th Street, \\ Omaha, Nebraska 68182-0681
}

Invited Papers

8:00

3aAAa1. Validation of an auralization system. Claus Lynge Christensen, Christoffer A. Weitze, Jens Holger Rindel, and Anders Christian Gade (Ørsted·DTU, Acoust. Technol., Tech. Univ. of Denmark, Bldg. 352, Ørsted Plads, 2800 Kgs. Lyngby, Denmark, clc@oersted.dtu.dk)

The room acoustics program ODEON provides auralization using fully filtered binaural room impulse responses, each reflection being filtered through nine octave bands and a set of head-related transfer functions. Using the full filtering scheme allows, in principle, a complete audible presentation of all the properties, time-variant frequency coloration, as well as directional information predicted by the room acoustics program. Two methods of verification have been applied. The first method is based on direct measurements on the impulse responses predicted by ODEON, using the room acoustics measuring system DIRAC in order to verify that the auralization method is actually capable of reproducing the predicted room acoustic parameters. Monaural auralization filters were used for this purpose. The other method is an audible comparison between in situ recordings of a singing person in real rooms and the ODEON auralization of the same situations. The latter verification is part of an ongoing European research project, CAHRISMA, on restoration of the acoustics in old Byzantine churches and mosques in Istanbul.

8:30

3aAAa2. Effect of model detail level on room acoustic computer simulations. David T. Bradley and Lily M. Wang (Univ. of Nebraska-Lincoln, Peter Kiewit Inst., 1110 S. 67th St., Omaha, NE 68182-0681, dbradley@mail.unomaha.edu)

Computer modeling of room acoustics is limited due to approximations regarding wavelength to surface size ratios and diffraction. In fact, it is suggested that a model with high geometrical fidelity may actually lead to less accurate predictions. This project seeks to clarify how the level of model detail affects the accuracy of these simulations. Three models of the University of Nebraska's Strauss Recital Hall have been generated at varying levels of detail; low (a rough model of the essential architectural dimensions), medium (a more accurate representation of the space), and high (a complete rendering of the surfaces within the space). The room acoustics modeling program Odeon is used to calculate objective measures (i.e., reverberation time and clarity) and create auralizations for each model. Meanwhile, the actual acoustical properties of Strauss Hall have been measured. The objective measure results are compared between the models and against measured data. Additionally, subjective tests are run with auralizations from all three models and the original space to determine if the difference in level detail can be perceived psychoacoustically. Quantitative results from these comparisons are presented to help users of room acoustic simulation software understand how the level of model detail will produce maximum accuracy.

9:00

3aAAa3. The development and use of the diffusion scattering coefficients in room modeling software: The effect of diffuse reflections on reverberation time. Peter D'Antonio (RPG Diffusor Systems, Inc., 651-C Commerce Dr., Upper Marlboro, MD 20774) and Trevor J. Cox (Univ. of Salford, Salford M5 4WT, UK)

In the last 20 years, much progress has been made in the measurement and characterization of sound diffusing surfaces. This presentation describes how this research might improve the accuracy of computer based geometric room modeling. To model diffuse reflections, one needs to know the scattering distribution and the portion of scattered energy that is diffusely reflected. AES Working Group SC-04-02 recently published an information document AES-4id-2001, which describes a measurement procedure to determine the polar balloons from scattering surfaces. This allows programmers to use actual measured or calculated distributions instead of Lamberts law, which applies to incoherent optical diffusion. ISO WG 25 is currently addressing a measurement procedure to determine the random incidence scattering coefficient. This scattering coefficient is used directly in geometrical modeling programs. The two measurement procedures will be contrasted. It will then be demonstrated by example how the use of diffusion data can affect the predicted reverberation time. 
3aAAa4. Uses and limitations of statistical and geometrical modeling of nonexponential decay curves in performance spaces. Jason E. Summers, Rendell R. Torres, Yasushi Shimizu (Prog. in Architectural Acoust., Rensselaer Polytechnic Inst., 110 8th St., Troy, NY 12180), and J. Christopher Jaffe (Jaffe Holden Acoust., 114A Washington St., Norwalk, CT 06854)

The conditions under which the high-frequency, diffuse-field model of coupled rooms is a valid approximation to geometrical acoustics have been examined by comparison with ray-tracing predictions of decay curves in two and three room systems. Results particular to coupled rooms were noted. Because nonexponential decay shape is sensitive to both decay rates and relative energy densities, corrections to these parameters meaningfully improved the diffuse-field model. Variations in the free-path distribution of each subroom, introduced by coupling, complicated the use of improved decay models. Also, the expected decrease in energy density with distance from the source was found to result in spatial dependence of decay shape for certain coupling geometries. Insights from this study were used in the construction of diffuse-field and geometrical computer models of Bass Hall, a 2056 seat multipurpose auditorium with an acoustically coupled stage house. Preliminary results indicate that high-frequency decay curves in each of the subrooms predicted by geometrical acoustics are well matched to the predictions of diffuse-field models. The use of both models as prediction and design tools is assessed by comparison at high frequencies with measurements made during occupied and unoccupied conditions. [Research supported by the Bass Foundation.]

\title{
10:00
}

3aAAa5. Case studies of computer model applications in consulting practice. Gary Siebein, Hyun Paek, Mark LoRang, and Courtney McGuinnes (Siebein Assoc., Inc., 625 NW 60th St., Ste. C, Gainesville, FL 32607)

Six case studies of computer model applications in a consulting practice will be presented to present the range of issues that can be studied with computer models as well as to understand the limitations of the technique at the present time. Case studies of elliptical conference rooms demonstrate basic acoustic ray principles and suggest remediation strategies. Models of a large themed entertainment venue with multiple amplified sound sources show how visualization of the acoustic ray paths can assist a consultant and client in value engineering locations and amounts of acoustic materials. The acoustic problems with an angled ceiling and large rear wall were studied when an historic church was converted to a music performance hall. The computer model of an historic hall did not present enough detailed information and was supplemented with physical model studies and full size mock-up tests of the insertion of an elevator door that would open directly into the concert room. Studies to demonstrate the amount of room model detail to obtain realistic auralizations were also conducted. The integration of architectural acoustic design and audio system design were studied in computer models of a large church sanctuary.

WEDNESDAY MORNING, 5 JUNE 2002

KINGS GARDEN SOUTH, 10:30 A.M. TO 1:00 P.M.

\section{Session 3aAAb}

\section{Architectural Acoustics: Student Design Competition}

\author{
Lily M. Wang, Cochair \\ Architectural Engineering, University of Nebraska, 200B Peter Kiewit Institute, 1110 South 67th Street, \\ Omaha, Nebraska 68182-0681 \\ Robert C. Coffeen, Cochair \\ School of Architecture and Urban Design, University of Kansas, Marvin Hall, Lawrence, Kansas 66045
}

The Technical Committee on Architectural Acoustics of the Acoustical Society of America and the National Council of Acoustical Consultants are sponsoring a student design competition which will be professionally judged at the meeting.

The purpose of this design competition is to encourage students enrolled in Architecture, Architectural Engineering, and other University curriculums that involve building design and/or acoustics to express their knowledge of architectural acoustics and building noise control in the schematic design of portions of a building where acoustical considerations are of primary importance.

The submitted designs, which will be displayed at the Pittsburgh ASA Meeting, will be judged by a panel of professional architects and acoustical consultants. An award of $\$ 1,000$ will be made to the submitter(s) of the design judged "first honors." Four awards of $\$ 500$ each will be made to the submitters of four entries judged "commendation." 


\title{
Session 3aABa
}

\section{Animal Bioacoustics: Acoustics of Terrestrial Mammals and Birds}

\author{
Ann E. Bowles, Chair \\ Hubbs-Sea World Research Institute, 2595 Ingraham Street, San Diego, California 92109
}

Chair's Introduction-8:00

Invited Papers

8:05

3aABa1. General principles involved in the effect of noise on hearing and vocal communication in birds. Robert J. Dooling and Michael L. Dent (Dept. of Psych., Univ. of Maryland, College Park, MD 20742, dooling @ psyc.umd.edu)

Birds provide very useful models for understanding the effects of noise on hearing and acoustic communication. They are excellent subjects for laboratory studies of hearing in which signals and noise can be precisely defined and delivered and behavioral responses can be unambiguously interpreted. For this reason, a huge amount is already known about their hearing. Acoustic communication is critically important for most species of birds and some even acquire their communication signals through vocal learning. For this reason, a lot is already known about how birds perceive complex acoustic signals such as vocalizations. Drawing from both field and laboratory studies, we review what is known about the effects of noise on hearing and vocal communication in birds. This includes the effects of intense noise on the ear, the effects of background noise on the detection and discrimination of both simple sounds and complex vocalizations, and the spatial effects of signal detection in noise in the free-field. As a whole, these studies show that birds are resistant to damage and interference from noise and have developed a variety of strategies to effectively communicate.

\section{8:25}

3aABa2. Analysis of auditory spatial receptive fields: An application of virtual auditory space technology. Terry T. Takahashi, Clifford H. Keller, David R. Euston, and Michael L. Spezio (Inst. of Neurosci., Univ. of Oregon, Eugene, OR)

Virtual auditory space technology, typically used to simulate acoustical environments, also allows one to vary one sound localization cue independently of others. VAST was used to determine the contributions of interaural time and level differences (ITD, ILD) to the spatial receptive fields (RFs) of neurons in the owl's midbrain. The presentation of noise filtered so that only ITD varied evoked a response along a vertical strip of virtual space, called the ITD-alone RF. Conversely, when ITD was fixed at the cell's optimum and the ILD spectrum of each location was presented, the cell responded along a horizontal strip, called the ILD-alone RF. The spatial RF was at the intersection of the ITD and ILD-alone RFs. The cell's ILD tuning across frequency, combined with individualized head-related transfer functions, was transformed into an ILD-alone RF that predicted half the variance in the measured one. This discrepancy was due partly to the poor response of the neurons to tones, and a new method of inferring frequency-specific ILD tuning from responses to noise explained about $75 \%$ of the variance. By understanding how spatial RFs are constructed, it is possible to infer the neural image of complex auditory scenes containing multiple sources and echoes. [Work supported by NIDCD.]

\section{8:45}

3aABa3. Sex differences in razorbill (Family: Alcidae) parent-offspring vocal recognition. Stephen J. Insley (Hubbs-Seaworld Res. Inst., 2595 Ingraham St., San Diego, CA 92109), Rosana Paredes Vela, and Ian L. Jones (Memorial Univ. of New Foundland, Canada)

In this study we examines how a pattern of parental care may result in a sex bias in vocal recognition. In Razorbills (Alca torda), both sexes provide parental care to their chicks while at the nest, after which the male is the sole caregiver for an additional period at sea. Selection pressure acting on recognition behavior is expected to be strongest during the time when males and chicks are together at sea, and as a result, parent-offspring recognition was predicted to be better developed in the male parent, that is, show a paternal bias. In order to test this hypothesis, vocal playback experiments were conducted on breeding Razorbills at the Gannet Islands, Labrador, 2001. The data provide clear evidence of mutual vocal recognition between the male parent and chick but not between the female parent and chick, supporting the hypothesis that parent-offspring recognition is male biased in this species. In addition to acoustic recognition, such a bias could have important social implications for a variety of behavioral and basic life history traits such as cooperation and sex-biased dispersal.

9:05

3aABa4. Identifying bird species from bird song using frequency component analysis. Jacky Mallett and Irene Pepperberg (Media Lab., MIT, 20 Ames St., Cambridge, MA 02139)

The species-distinctive and highly stereotyped nature of bird vocalizations makes birdsong an obvious candidate for computational analysis. By using techniques from previous work in automatic music recognition and auditory analysis, a database of the detailed auditory characteristics of a selection of songs from over 150 different birds from the North Eastern region of the United States of America has been created. Examination of this database has allowed a picture to be built up of species-distinctive characteristics, and 
provides a more general picture of bird vocalizations than has previously been available. From this work it appears that frequency use by birds is of some significance, with evidence that the presence of a particular set of dominant frequencies in a bird's song can be used as a distinctive fingerprint for species recognition. This paper reviews previous work in this area, outlines general computational methods used to extract this information, and presents results of automatic bird recognition using characteristics derived from the database.

$9: 25$

3aABa5. Acoustic surveys for Mexican spotted owls (Strix occidentalis lucida): An analysis. Ann E. Bowles, Chris Martindell (Hubbs-Sea World Res. Inst., 2595 Ingraham St., San Diego, CA 92109, annb1@san.rr.com), Kenneth J. Plotkin, Bruce Ikelheimer (Wyle Labs., Ste. 701, 2001 Jefferson Davis Hwy., Arlington, VA 22202), and Tim Lavallee (GeoMarine, Inc., 11846 Rock Landing Dr., Newport News, VA 23606)

During acoustic surveys for Mexican spotted owls, the effective detection range is presumed to be $0.25 \mathrm{mi}(433 \mathrm{~m})$. However, variations within and between surveys are observed, leading to a variance in owl density estimates. While owl behavior may explain some variation, topography and ambient noise are also likely to be important. To determine the influence of these factors, data from acoustic surveys for Mexican spotted owls in the Gila National Forest (April-July, 2000-2001) were examined. Measurements of owl and human call levels were made with a Sony TCD-10 Pro II DAT equipped with an ACO 7013 microphone. Ambient noise was collected using 40 Larson-Davis 820 and 824 sound level meters in owl activity centers. Wyle Laboratories NMSIM software was used to model propagation of owl and human calls. Owls produced calls with estimated maximum source levels of 92-98-dB SPL. Human callers produced maximum source levels of $88-95-\mathrm{dB}$ SPL. Detection was possible out to more than $2 \mathrm{~km}$ under ideal conditions, but topography and ambient noise had a large effect. Corrections for these factors would greatly improve estimates of area surveyed, and thus owl density estimates. [Work supported by the U.S. Air Force, Air Combat Command.]

\section{9:45-9:55 Break}

\section{Contributed Papers}

\section{9:55}

3aABa6. Frequency selectivity in canaries with a hereditary hearing loss. Amanda M. Lauer and Robert J. Dooling (Dept. of Psych., Univ. of Maryland, College Park, MD 20742, alauer@psyc.umd.edu)

Sensorineural hearing loss is associated with reduced frequency selectivity due to the broadening of the auditory filters in mammals. In European starlings, hearing loss caused by the ototoxic drug kanamycin results in the widening of auditory filters at $5 \mathrm{kHz}$ [Marean et al. (1998)]. In the present study, we examine frequency selectivity in a bird with a permanent hereditary hearing impairment, the Belgian Waterslager (BWS) canary. This strain of canary has long been bred for its loud, low-frequency song, and has been shown to have a hearing loss primarily at higher frequencies ( $2 \mathrm{kHz}$ and above). Using operant conditioning and the method of constant stimuli, thresholds for detecting pure tones in flat-spectrum broadband noise were measured in BWS and non-BWS canaries. Critical ratios were calculated for comparison with other species of birds. At higher frequencies, critical ratios for BWS canaries were much larger than those of non-BWS canaries and other birds, suggesting reduced frequency selectivity in the region of the birds' hearing loss. [Work supported by NIDCD R01DC001372 to RJD and Brenda M. Ryals.]

\section{0:10}

3aABa7. Narrowband masking in birds. Stacey L. Brown, Robert J. Dooling (Dept. of Psych., Univ. of Maryland, College Park, MD 20742, sbrown@psyc.umd.edu), and Marjorie R. Leek (Walter Reed Army Medical Ctr., Washington, DC 20307)

Hearing studies show that zebra finches, Taeniopygia guttata, small song birds that produce broadband, harmonic vocalizations are unusually sensitive to the mistuning of a single component in a harmonic complex [Lohr and Dooling, J. Comput. Psychol. 112, 36-47 (1998)]. Such a change in a harmonic stimulus creates both spectral and temporal cues. Previously it has been reported that these birds have very good temporal resolution of fine detail within harmonic complexes [Dooling et al., Hear. Res. 152, 159-172 (2001)]. Here the spectral resolving power is examined by measuring auditory patterns of masking by narrowband noises. Masking patterns were measured in zebra finches using operant conditioning and the method of constant stimuli for a $100-\mathrm{Hz}$ band noise masker centered at the birds frequency of best hearing, $2860 \mathrm{~Hz}$. Probe frequencies were tones between $1000 \mathrm{~Hz}$ and $5700 \mathrm{~Hz}$. Results demonstrate a symmetrical masking pattern with less upward spread of masking and a nar- rower resolution bandwidth in zebra finches than has been observed in humans. These findings are consistent with other measures of avian spectral resolution. [Work supported by NIH Grant DC-00198 to RJD.]

\section{$10: 25$}

3aABa8. Computer synthesis of bird songs and calls. Mark Kahrs (Dept. of Elec. Eng., Univ. of Pittsburgh, 348 Benedum Hall, Pittsburgh, PA 15261) and Federico Avanzini (Universitá degli Studi di Padova, 35131 Padova, Italy)

We describe the computer simulation of a one-mass source together with a simple transmission line model for a psittacine bird. The syrinx is modeled as a lumped mass subject to elastic restoring forces and internal dissipation. Nonlinear interaction with the airflow follows the Ishizaka and Flanagan glottal model. This is also used to model complete closure of the syrinx: during closure an additional restoring force is added and dissipation is increased. During the whole closed phase the syringeal flow is zero, and consequently its spectrum is broadened and higher partials are generated. The vocal tract transmission lines are implemented with fractional delay lines and the transmission lines are assumed lossless. The beak is implemented as two complementary fifth order Butterworth filters. The pole trajectory for the filters approximates the nonlinear path of the beak cutoff as discussed by Fletcher. [M.K. was supported by the Fulbright Foundation as well as Tekes. F.A. was funded by the Sound Source Modeling Project. Both authors were supported in part by the Academy of Finland.]

\section{0:40}

3aABa9. Discrimination of dynamic moving ripples in the zebra finch, Taeniopygia guttata. Michael S. Osmanski, Robert J. Dooling (Dept. of Psych., Univ. of Maryland, College Park, MD 20742), and Didier A. Depireux (Univ. of Maryland at Baltimore, Baltimore, MD)

Auditory processing of complex broadband sounds known as moving ripples has been studied both physiologically at the cortical level in mammals and psychophysically in humans [Depireux et al. (2001); Chi et al. (1999)]. These stimuli share spectro-temporal properties with many natural sounds, including species-specific vocalizations and the formant transitions of human speech [Versnel and Shamma (1998)]. One test of the generality of ripple processing beyond mammals would be to examine a non-mammalian species. Zebra finches may be excellent subjects for such 
a study because they produce complex broadband harmonic songs and neuronal responses in their auditory forebrain may be exquisitely tuned to the specific spectro-temporal patterns of their songs [Theunnissen and Doupe (1998)]. We trained these birds to discriminate between flatspectrum broadband noise and moving ripples of different densities that move up or down in frequency at various rates. Results show that discrimination in zebra finches is better at those ripple densities and velocities which are prominent in their species-specific harmonic vocalizations. [Work supported by NIH Grant No. DC-00198 to RJD and NIDCD Training Grant No. DC-00046.]

WEDNESDAY MORNING, 5 JUNE 2002

LE BATEAU ROOM, 10:55 A.M. TO 12:15 P.M.

\title{
Session $3 a A B b$
}

\section{Animal Bioacoustics: General Topics in Animal Bioacoustics}

\author{
Ann E. Bowles, Chair \\ Hubbs-Sea World Research Institute, 2595 Ingraham Street, San Diego, California 92109 \\ Chair's Introduction-10:55
}

Contributed Papers

11:00

$11: 30$

3aABb1. Acoustical and functional analysis of Mountain lion (Puma concolor) vocalizations. Jacquelyn Potter (Dept. of Biological Sci., Western Illinois Univ., 1 University Circle, Macomb, IL 61455, jacquelyn_potter@yahoo.com)

A 2-year study resulted in acoustic analysis of the structure of over 900 mountain lion vocalizations recorded in a seminatural setting at Wildlife Prairie Park near Peoria, Illinois. A vocal repertoire was obtained by describing quantitative variables about the sounds, i.e., frequency of the dominant part of the sound (beginning, ending, maximum, and minimum), duration, and number of components. Other variables described the tonal, harmonic, and wideband qualities of the sounds. Behavioral data were collected during the same period. Further analysis of both acoustic and behavioral data was completed to develop a correlation matrix between vocalizations and behavior. This study also looked at the effects of seasons on vocal behavior. Correlations were found between vocalization types and rates of usage with specific behaviors. Vocalization type and the usage rate also varied by season.

\section{1:15}

3aABb2. Acoustic correlates of human responses to domestic cat (feliscatus) vocalizations. Nicholas Nicastro (Dept. of Psych., Cornell Univ., 243 Uris Hall, Ithaca, NY 14853)

As part of ongoing research on coevolution of vocal communication between humans and domestic cats, perceptual data were collected on participants as they listened to recorded cat vocalizations. In experiment 1 , human subjects were asked to rate the pleasantness of 100 meows along a 7-point scale, from most to least pleasant. In experiment 2, a different group of participants was asked to rate the urgency of the same meows along a 7-point scale, from most to least urgent. Linear regression analysis of the results showed a strong inverse correlation between pleasantness and urgency. Acoustic correlates of pleasantness included reduced frequency modulation, a downward shift in fundamental frequency, and fewer noisy segments. Correlates of urgency included increased duration, higher Wiener entropy, and acute spectral tilt. It is speculated that humans' affective responses to these acoustic qualities, in conjunction with contextual cues, may form the basis of the communication of more specific meanings by cats to humans.
3aABb3. Extraction of directional pitch change by Japanese monkeys. Tokuro Takahashi and Hiroshi Riquimaroux (Dept. of Knowledge Eng. \& Computer Sci., Doshisha Univ., Kyotanabe, Kyoto 610-0321, Japan)

Perception of directional pitch change was behaviorally investigated in Japanese monkeys on an operant Go-Nogo task with water reward. Results show that the difference in the fundamental frequency makes a difference in direction of pitch change. A pair of tone bursts and/or harmonically structured complex tone bursts were sequentially presented. The monkeys could always discriminate the direction of pitch change by using the fundamental frequency cue but in a specific case. Their performance was worse than a chance level, or they oppositely responded to the fundamental frequency cue in the specific case. The case was that the temporal sequence was made of a simple tone burst and a complex tone burst where the frequency of the simple tone burst was in between the fundamental and the second harmonic frequencies of the complex tone burst, and the fundamentals of the complex tone were lower than $500 \mathrm{~Hz}$. They showed amelioration in using the fundamental frequency cue as interval between two tones was expanded. The results were confirmed with probe tests. [Research supported by Special Coordination Funds, and a grant to RCAST at Doshisha University from the Ministry of Education, Culture, Sports, Science and Technology of Japan.]

\section{$11: 45$}

3aABb4. Sound duration as a perceptual cue influencing vocal behavior of male bullfrogs. Andrea M. Simmons (Dept. of Psych., Brown Univ., Providence, RI 02912, Andrea_Simmons@brown.edu)

Female frogs of several species use the temporal cue of sound duration to aid in mate choice. Little is known, however, about the sensitivity of male frogs to this cue. Male bullfrogs emit a complex advertisement call to attract females for mating, and to announce territory occupation to other males. In two experiments, the sensitivity of vocalizing male bullfrogs to field playbacks of advertisement calls differing in duration was examined. The number and latency of evoked vocal responses to the stimuli was used as a measure of perception. Males responded with fewer calls, at longer latencies, to stimuli shorter in duration than the standard signal (with a duration at the mean value for the species). Males preferred stimuli longer in duration than the standard signal, responding with more calls at shorter latencies. They did not, however, significantly lengthen their own calls in response to playbacks of long duration signals. This preference for "supernormal" stimuli may be an important factor mediating the evolution of communication signals. [Work supported by NIH.] 
nonexistent role. However, it can be shown that acoustic communication between ants is effective only if the medium is air and not the substrate. How, then, is it possible for ants to appear deaf to airborne sound and yet communicate through the air? An explanation is provided in a paper [R. Hickling and R. L. Brown, "Analysis of acoustic communication by ants," J. Acoust. Soc. Am. 108, 1920-1929 (2000)]. Ants are small relative to the wavelengths they generate. Hence, they create a near field, which is characterized by a major increase in sound velocity (particle velocity of sound) in the vicinity of the source. Hair sensilla on the ants' antennae respond to sound velocity. Thus, ants are able to detect near-field sound from other ants and to exclude extraneous airborne sound.

WEDNESDAY MORNING, 5 JUNE 2002

RIVERS ROOM, 8:45 TO 11:00 A.M.

\title{
Session 3aMU
}

\section{Musical Acoustics: General Topics in Musical Acoustics}

\author{
Uwe J. Hansen, Chair \\ Department of Physics, Indiana State University, Terre Haute, Indiana 47809
}

\section{Contributed Papers}

\section{$8: 45$}

3aMU1. The effect of inharmonic partials on pitch of piano tones. Brian E. Anderson (Dept. of Phys., Brigham Young Univ., Provo, UT 84602, loudspeakerdesign@ @otmail.com)

Piano tones have partials whose frequencies are sharp relative to harmonic values. A listening test was conducted to determine the effect of inharmonicity on pitch for piano tones in the lowest three octaves of a piano. Nine real tones from the lowest three octaves of a piano were analyzed to obtain frequencies and amplitude of their partials. Synthetic inharmonic tones were produced from these results. Synthetic harmonic tones, each with a twelfth of a semitone increase in the fundamental, were also produced. A jury of 10 listeners matched each synthetic inharmonic tone to one of the synthetic harmonic tones. The effect of the inharmonicity on pitch was determined from an average of the listeners' results. For the nine piano tones studied, inharmonicity increased pitch from a twelfth of a semitone to nearly a semitone.

\section{9:00}

3aMU2. Prosodic persistence in music performance and speech production. Melissa K. Jungers, Caroline Palmer (Dept. of Psych., Ohio State Univ., 1885 Neil Ave., Columbus, OH 43210, jungers.2@osu.edu), and Shari R. Speer (Ohio State Univ., Columbus, OH 43210)

Does the rate of melodies that listeners hear affect the rate of their performed melodies? Skilled adult pianists performed two short melodies as a measure of their preferred performance rate. Next they heard, on each trial, a computer-generated performance of a prime melody at a slow or fast rate (600 or $300 \mathrm{~ms}$ per quarter-note beat). Following each prime melody, the pianists performed a target melody from notation. The prime and target melodies were matched for meter and length. The rate of pianists' target melody performances was slower for performances that followed a slow prime than a fast prime, indicating that pianists' performances were influenced by the rate of the prime melody. Performance duration was predicted by a model that includes prime and preferred du- rations. Findings from an analogous speech production experiment show that a similar model predicts speakers' sentence rate from preferred and prime sentence rates. [Work supported by NIMH Grant 45764 and the Center for Cognitive Science.]

\section{9:15}

3aMU3. Sound of a cup with and without instant coffee. Andrew Morrison and Thomas D. Rossing (Phys. Dept., Northern Illinois Univ., DeKalb, IL 60115)

An empty coffee cup, like an ancient Chinese two-tone bell, emits two distinctly different tones, depending upon where it is tapped. When it is filled with hot water, and some instant coffee is added, however, a whole new set of sounds is heard when the cup is tapped. The pitch rises an octave or more as the foam clears due to the dramatic change in the speed of sound in the bubble-filled liquid. A similar, but smaller, effect was noted in beer by Bragg [The World of Sound (1968)] and in hot chocolate by Crawford [Am. J. Phys. (1982)]. We describe the modes of vibration in a coffee cup and the sound emitted by a coffee cup as filled with instant coffee as the bubble density changes.

\section{$9: 30$}

3aMU4. Computer-animated illustrations of vibrations and waves. W. Bruce Richards (Oberlin College, Oberlin, OH 44074)

Undergraduate students in a general-education course on musical acoustics often have difficulty understanding the actual time-domain motions of vibrating systems which textbooks can only depict using static diagrams. In particular, students have difficulty relating the longitudinal average displacements of gas molecules in acoustic waves to (transverse) graphs of these displacements as a function of position. To better convey these ideas, a number of animated graphic diagrams have been programmed on a Macintosh computer. For longitudinal waves, these include 
one-dimensional standing waves in cylindrical pipes with open or closed ends, various pulse waves reflecting from the open or closed end of a cylindrical pipe, and standing waves in a conical pipe with an open end. In addition, the transverse motions of bowed, plucked, and hammered strings have been programmed. Presented in every case is an exaggerated, slow motion picture of the system's displacement as it evolves in time. If desired, an adjacent coordinated and animated graph of displacement, pressure, or other dynamical variable may also be displayed. As part of this paper the computer programs will be demonstrated.

\section{$9: 45$}

3aMU5. Drumhead contact time measurement using metallic leaf. José Sánchez and James Irwin, Jr. (Dept. of Elec. \& Computer Eng., 1501 W. Bradley Ave., Bradley Univ., Peoria, IL 61625, jhirwin@bradley.edu)

This work investigates the relationship between mass, diameter, impact location, and energy of steel balls impacting upon the surface of the drumhead, and the time they remain in contact. The contact time is measured by making the ball and metallic leaf contacts in an electrical switch. The leaf was applied to the drumhead surface and connected to the circuit through a wire. The balls were connected to the circuit using a solderattached hair-fine wire. Contact time was measured at a constant drumhead tension at three distinct locations on the drumhead: the center, halfway between the center and the rim, and an inch from the rim. In addition, a single series was measured from the center to the rim at $\frac{1}{2}$-in. intervals. Initial work indicates that the contact time is not dependent upon energy, impact location, or diameter, but only upon the mass of the steel ball. The relationship of the mass-dependent contact time to energy transfer and to impact spectra is developed, showing mass is the determining parameter needed for the initial conditions used to simulate drumstick/drumhead interaction (impact effects).

\section{0:00}

3aMU6. Mode studies in triangular bellplates. Uwe J. Hansen (Dept. of Phys., Indiana State Univ., Terre Haute, IN 47803), Daniel Lavan, and Suzanne Hogg (Tech. Univ., Sydney, Australia)

Preliminary results from mode studies in triangular aluminum bellplates using electronic holographic interferometry were reported at the 136th ASA meeting [Uwe J. Hansen, J. Acoust. Soc. Am. 104, 1767(A) (1998)]. Since then the TU Sydney co-authors of this paper have performed finite element calculations with results which are remarkably congruent with the holographic data. The first 13 modes will be discussed and shown in holographic as well as FEM representation.

\section{0:15}

3aMU7. Shape of a clamped stiff harpsichord wire driven at a resonant frequency. Roger J. Hanson, Hilliard Kent Macomber, and Mathew A. Boucher (Dept. of Phys., Univ. of Northern Iowa, Cedar Falls, IA 50614, roger.hanson@cfu.net)

A wire transversely driven by a sinusoidal force at the resonant frequency of a vibrational mode vibrates at the driving frequency and at harmonics generated by nonlinear processes in the wire. If the amplitude of a harmonic is measured as a function of position along the wire, its shape is revealed. It differs significantly from a sinusoid in the vicinity of either end of the wire because the ends are clamped and the wire has significant stiffness. The shapes of various harmonics have been determined for a brass harpsichord wire, $70 \mathrm{~cm}$ long, from optical detector measurements made at different distances from a clamped end. Knowledge of shape facilitates the determination of antinode amplitudes of harmonics when the gross motion of the wire is so large that the detectors must be positioned near an end of the wire because of their very limited dynamic range. Some observations of harmonics and related phenomena were reported previously [Hanson et al., J. Acoust Soc. Am. 108, 2592 (2000); 106, 2141 (1999)]. The shape information is also needed to help separate nonlinear effects possibly occurring in the detectors from those of interest, occurring in the wire itself.

\section{0:30}

3aMU8. Regimes of oscillation and reed vibrations in lingual organ pipes. Eric Cox and Thomas D. Rossing (Phys. Dept., Northern Illinois Univ., DeKalb, IL 60115)

We compare the sound generation in six lingual organ pipes including two trompette pipes, two oboes pipes, a krummhorn, and a rankett, the latter two of which were described in a previous paper [Cox and Rossing, paper 2aMU8, 142nd ASA meeting]. With the resonator attached, each pipe sounds in distinct regimes of oscillation, separated by discontinuities. The nominal frequencies of the pipes are generally slightly lower than the resonance frequency of the resonator but slightly higher than the natural frequency of the reed. The air mass loading of both the resonator and shallot lowers the reed frequency. Changing the blowing pressure has little effect on the sounding frequency but a modest effect on sound level.

10:45

3aMU9. Acoustic analysis of an Olmecan whistle. Sergio Beristain (President IMA, Lab. Acoust., Faculty of Elec. and Mech. Eng., Natl. Polytechnic Inst., P.O. Box 75805, 07300 Mexico City, Mexico), Rolando Menchaca (ESCOM, IPN), and Roberto Velazquez (CIC, IPN)

Thousands of stone artifacts over 2500 years of age have been found in the Olmecan area in the southeast region of Mexico. These range from the famous big heads with helmets (about $2 \mathrm{~m}$ in diameter), to small pieces with precisely drilled holes, which some archaeologists consider to have some simple uses, much simpler than the work needed to produce the stone artifact itself. The one studied here (about $3 \mathrm{~cm}$ in size), is considered by the acoustics community as an air-phone, and a detailed anaysis has been done employing FFT techniques in order to find out the frequency spread, the particular tones produced and the sound radiated power through the different holes and cavities. The artifact is made of a very solid stone, so-called ilmenite, believed to have titanium, which is very hard to drill. Nevertheless, many similar pieces have been found in the area, which means they were built on purpose, and the material used gives the idea of a sacred application. Attempts have been made to reproduce the artifacts, which produce sounds similar to those from the original pieces. 


\title{
Session 3aNS
}

\section{Noise, Speech Communication and Animal Bioacoustics: Public Policy for Noise}

\author{
Lawrence S. Finegold, Chair \\ Finegold \& So, Consultants, 1167 Bournemouth Court, Centerville, Ohio 45459
}

Invited Papers

\begin{abstract}
8:00
3aNS1. Public policy and the ASA. E. J. Walsh (Boys Town Natl. Res. Hospital, Omaha, NE 68131)

At the Ft. Lauderdale meeting of the Acoustical Society of America, the President of the Society authorized the formation of an ad hoc public policy committee to be named the Panel on Public Policy (PoPP). The Panel's mission is to serve the public interest by addressing and commenting on societal issues that fall within the broad range of disciplines that define the scope of the Society. The Panel's goal is to guide the development of ASA policies on, and responses to, questions that arise in relation to those issues. The operational plan calls for the Panel to execute its responsibility by generating and releasing Executive Council mandated and approved position statements, reports, or results of in-depth studies on issues affecting public health and welfare for use by appropriate legislative and policy-making entities. The structure, composition, and operational plan of the Panel will be discussed in general terms and specific prospective projects will be summarized.
\end{abstract}

8:10

3aNS2. Rationale for noise regulation: How we got here and why we may not stay. Sanford Fidell (Fidell Assoc., Inc., 23139 Erwin St., Woodland Hills, CA 91367)

Today's framework of environmental noise regulation in the United States is the product of a half century's evolution in understandings of noise effects, and in societal interest and policy interpretations of them. Although it is tempting to associate the evolution of noise policy with rational adaptations to developments in scientific understanding, this is a myopic and ultimately untenable view. It is hardly surprising that little of practical utility for regulatory purposes has come from decades of research in this area, considering the unsystematic and atheoretical nature of much of what has been learned about individual and community response to noise. Today's annoyance-based rationale for regulatory policy is only one of several historical perspectives, and not necessarily the most useful of them. Further changes in the rationale for assessing noise impacts may be expected as court rulings increasingly challenge reliance upon long-term exposure metrics as predictors of noise effects; as the magnitude of non-acoustic contributions to annoyance becomes clearer; and as the information that the U.S. Federal Interagency Committee on Noise last reviewed a decade ago becomes more and more stale.

8:40

3aNS3. National policy on the effects of underwater noise on marine mammals and turtles. Roger L. Gentry (NOAA, Natl. Marine Fisheries Service, Silver Spring, MD 20910)

Marine anthropogenic noise is increasing at an unknown rate yet its effects on mammals and turtles are poorly documented. Such information is central to noise standards for marine animals required by two federal laws (MMPA and ESA). Good policy on underwater noise would set standards that are neither too liberal (thereby threatening the well being of marine animals), nor too conservative (thereby unnecessarily impairing essential human activities at sea). This balance must be found using existing data; standards cannot await new research. Policy must also lay out rules governing the introduction of novel underwater sound sources whose effects on animals are unknown. Should policy forbid such introductions until all possible consequences are known, or does a safe, incremental method of introduction exist? Should exceptions be made for novel sources associated with national defense? Policy must cope with sound sources that are outside the reach of U.S. federal regulations, such as international shipping. Finally, policy must frame the questions about underwater noise in a way that research can answer. Specifically, how much noise exists, how fast is it growing, where is it, and what consequences does it have for animals and their ecosystems?

9:00

3aNS4. Determination of background sound level for use with Massachusetts community sound level criteria. Gregory Tocci (Cavanaugh Tocci Assoc., Inc., 327F Boston Post Rd., Sudbury, MA 01776)

In the early 1970s, the Commonwealth of Massachusetts began the use of their community sound level criteria. In 1990, these criteria were formalized as Policy 90-001. The policy states that: A source of sound will be considered to be violating the Department's noise regulation (310 CMR 7.10) if the source increases the broadband sound level by more than $10 \mathrm{~dB}(\mathrm{~A})$ above the ambient. Ambient is defined as the background A-weighted sound level that is exceeded $90 \%$ of the time measured during equipment operating hours. The Policy also address tonal sound, and this will be described, but the primary concern of this presentation is the measurement of background sound. A number of background sound measurement strategies will be discussed and a recommendation made on how background should be measured and analyzed that is easily understood by regulators, developers, and the community. 
State and local governments have adopted a variety of regulations over the past 40 years to protect the public against unreasonable noise. These regulations, which may be be qualitative in nature, may prohibit particular activities (e.g., barking dogs or loud radios) or outlaw nuisance noise in general (e.g., sounds disturbing to a resident). In addition, these regulations may be quantitative and define specific sound limits for a source at property line or community locations. The quantitative approach may typically include absolute limits that account for many factors, such as the time of day or week, the type of source and receptor, and the character of the sound; or in the case of Massachusetts, may include relative limits that account for the ambient background sound levels. A significant increase in community sound levels that is caused by a source is a useful indicator of a potential noise problem, but should this factor be considered when setting regulatory limits for individual projects? The presentation shall explore this issue and its effect on the process of siting, permitting, designing, and operating industrial facilities.

\section{9:40}

3aNS6. The need to revitalize our national noise policy. William W. Lang (29 Hornbeck Ridge, Poughkeepsie, NY 12603, langww@alum.mit.edu) and Leo L. Beranek (Cambridge, MA 02138)

At the present time, our Nation does not have a national noise policy. The Congress attempted to define one with the passage of the Noise Control Act of 1972 as follows: The Congress declares that it is the policy of the United States to promote an environment for all Americans free from noise that jeopardizes their health and welfare. The Act assigned to the Environmental Protection Agency (EPA) the role of leading Federal agency with the task of coordinating the programs of all Federal agencies relating to noise research and noise control. Ten years later (1982), all funds for noise control were withdrawn from the EPA, and today the Nation is without an effective, overall noise policy. Residual responsibility for the control of environmental and occupational noise currently rests with a dozen agencies of the Federal government, as well as State, municipal, and local authorities. But the activities of these organizations are largely uncoordinated, and the enforcement of existing noise control regulations is at best sporadic, and in some cases nonexistent. To assure our quality of life and to minimize the economic impact of potential trade barriers, a study team is recommending that a national noise policy be developed and then implemented in the immediate future.

10:00-10:15 Break

\section{0:15}

3aNS7. Revisiting the NIOSH Criteria for a Recommended Standard: Occupational Noise Exposure. William J. Murphy and John R. Franks (Natl. Inst. for Occupational Safety and Health, Hearing Loss Prevention Section, 4676 Columbia Pkwy., M.S. C-27, Cincinnati, OH 45226-1998, wjm4@cdc.gov)

In 1998, the National Institute for Occupational Safety and Health (NIOSH) revised the Criteria for a Recommended Standard: Occupational Noise Exposure [DHHS (NIOSH) Publication No. 98-126]. NIOSH reevaluated the recommended exposure limit (REL) for occupational noise exposure and reaffirms support for 85-dBA REL. Based upon scientific evidence, NIOSH recommends a 3-dB exchange rate. NIOSH recommends that significant threshold shift be identified as an increase of $15 \mathrm{~dB}$ in the hearing threshold level at $500,1000,2000,3000,4000$, or $6000 \mathrm{~Hz}$ in either ear, with two consecutive audiometric tests. The new criterion has the advantages of a high identification rate and a low false-positive rate. In contrast with the former 1972 criterion, NIOSH no longer recommends age correction on individual audiograms. NIOSH has revisited its recommendations on the using of single-number laboratory-derived Noise Reduction Rating (NRR) required by the U.S. Environmental Protection Agency for labeling of hearing protectors sold within the United States. In 1972, NIOSH recommended the use of the full NRR value; however, the new criterion recommends derating the NRR by $25 \%, 50 \%$, and $70 \%$ for earmuffs, formable earplugs, and all other earplugs, respectively. This presentation will compare and contrast current regulations against the NIOSH recommendations.

\section{0:35}

3aNS8. Benefits and disadvantages of self-regulation of environmental noise from military training. George A. Luz (U.S. Army Ctr. for Health Promotion and Preventive Medicine, 5158 Blackhawk Rd., Aberdeen Proving Ground, MD 21010-5403)

In a 1981 Executive decision, the Administration's Office of Management and Budget (OMB) told the Environmental Protection Agency to end funding of the Office of Noise Abatement and Control (ONAC). This decision, coupled with a specific exemption for military equipment contained in the Noise Control Act of 1972, ensured that the military departments would be self-regulating in regard to noise. This self-regulation for noise stands in contrast to the external regulation of other pollutants, such as air and water emissions. Two possible disadvantages of self-regulation are (1) reduced funding for noise management compared with funding for externally regulated pollutants, and (2) lack of an independent and external set of standards for determining acceptable limits on community noise exposure. Three possible benefits are (1) avoiding the costs of mitigating trivial violations of external standards, (2) maintaining a long-standing policy of preventing noise problems through land use planning, and (3) enabling negotiated solutions between installations and their neighboring communities. The paper ends with an examination of a negotiated solution for a community subjected to noise from the detonation of obsolete ammunition. 
3aNS9. Public policy issues in animal bioacoustics. Ann E. Bowles (Hubbs-Sea World Res. Inst., 2595 Ingraham St., San Diego, CA 92109) and Mardi C. Hastings (Biomed. Eng. Ctr., Ohio State Univ., 1080 Carmack Rd., Columbus, OH 43210)

Control of anthropogenic noise in many terrestrial and underwater environments is crucial for maintaining communication, health, and normal behavior of animals. Noise can be an issue for any species; usually, however, endangered and threatened species and marine mammals are the ones provided legal protection under the Endangered Species Act and/or the Marine Mammal Protection Act. Recent substantiated noise effects in the ocean have received much publicity and generated a more global approach to noise control. However, there are also cases where publicity was not accompanied by scientific data substantiating effects (e.g., an incident involving a $\$ 1$ million noise barrier installed to protect passerine birds). The public and environmental managers have had difficulty developing adequate guidelines not only because necessary data are often lacking, but also because the manner in which funding is allocatednoise-producing agencies or private organizations are often pressured to fund studies—gives rise to inevitable conflicts of interest (or the perception thereof). Examples of recent noise-related controversies will be presented to examine the role of scientists, engineers, and professional organizations such as ASA in dealing with conflicts of interest and formulating public policy.

\title{
11:15
}

3aNS10. Community-based environmental noise management. Lawrence S. Finegold (Finegold \& So, Consultants, 1167 Bournemouth Ct., Centerville, OH 45459, LSFinegold@earthlink.net)

After a series of major successes in implementing national environmental noise policies from the 1960s to the 1980s, the U.S. has not kept up with improvements in environmental noise policies seen in other areas of the world, most notably in Europe during the past decade. The cessation of funding for the EPA Office of Noise Abatement and Control (ONAC) in 1981 was a defining point in the history of U.S. environmental noise policy, leading to a virtual stoppage of the development of new noise regulations. The Noise Control Act (NCA) of 1972, which established EPA ONAC, was never rescinded after ONAC funding was abolished, but it is not being implemented either. Thus national noise policies are generally not being enforced, except for aircraft certification regulations, and help for state and local efforts is inadequate. In this paper we describe an evolving concept, community-based environmental noise management, to provide practical guidance to local communities in developing noise management strategies. This concept includes components such as local noise ordinances, coordination with State and Federal agencies, incentive and cost-sharing programs, land use planning, an updated environmental impact analysis process, and a negotiation and dispute resolution process. Each of these will be briefly discussed.

WEDNESDAY MORNING, 5 JUNE 2002

STERLINGS ROOMS 2 AND 3, 8:00 TO 10:00 A.M.

\section{Session 3aPA}

\section{Physical Acoustics: Surface Waves and Ultrasound Spectroscopy}

\author{
Joseph R. Gladden, Cochair \\ Physics Department, Pennsylvania State University, 104 Davey Laboratory, University Park, Pennsylvania 16802 \\ Ralph T. Muehleisen, Cochair \\ Civil, Environmental and Architectural Engineering, University of Colorado, 428 UCB, 441 Ecot, \\ Boulder, Colorado 80309-0428
}

\section{Contributed Papers}

\section{8:00}

3aPA1. Acoustically excited surface waves on empty or fluid-filled cylindrical and spherical shells. A. Claude Ahyi, H. Cao, P. K. Raju (Dept. of Mech. Eng., Auburn Univ., Auburn, AL 36849-5341), M. F. Werby (Dept. of Phys., Catholic Univ. of America, Washington, DC 20064-1 and NRL Code 7180, Stennis Space Center, MS 39529), X. L. Bao, and H. Uberall (Dept. of Phys., Catholic Univ. of America, Washington, DC 20064-1)

A comparative study is presented of the acoustical excitation of circumferential (surface) waves on fluid-immersed cylindrical or spherical metal shells, which may be either evacuated, or filled with the same or a different fluid. The excited surface waves can manifest themselves by the resonances apparent in the sound scattering amplitude, which they cause upon phase matching following repeated circumnavigations of the target object, or by their re-radiation into the external fluid in the manner of head waves. We plot dispersion curves versus frequency of the surface waves, which for evacuated shells have a generally rising character, while the fluid filling adds an additional set of circumferential waves that descend with frequency. The resonances of these latter waves may also be interpreted as being due to phase matching, but they may alternately be interpreted as constituting the eigenfrequencies of the internal fluid contained in an elastic enclosure.

\section{$8: 15$}

3aPA2. Acoustic resonances of fluid-immersed elastic cylinders and spheroids: Theory and experiment. Jan Niemiec (Naval Surface Warfare Ctr., Carderock Div., West Bethesda, MD 20817-5700), Herbert Überall, and X. L. Bao (Catholic Univ. of America, Washington, DC 20064-1)

Frequency resonances in the scattering of acoustic waves from a target object are caused by the phase matching of surface waves repeatedly encircling the object. This is exemplified here by considering elastic finite cylinders and spheroids, and the phase-matching condition provides a means of calculating the complex resonance frequencies of such objects. Tank experiments carried out at Catholic University, or at the University of Le Havre, France by G. Maze and J. Ripoche, have been interpreted 
using this approach. The experiments employed sound pulses to measure arrival times, which allowed identification of the surface paths taken by the surface waves, thus giving rise to resonances in the scattering amplitude. A calculation of the resonance frequencies using the T-matrix approach showed satisfactory agreement with the experimental resonance frequencies that were either measured directly (as at Le Havre), or that were obtained by the interpretation of measured arrival times (at Catholic University) using calculated surface wave paths, and the extraction of resonance frequencies therefrom, on the basis of the phase-matching condition. Results for hemispherically endcapped, evacuated steel cylinders obtained in a lake experiment carried out by the NSWC were interpreted in the same fashion.

\section{8:30}

3aPA3. Experimental study of the A-wave axial propagation inside a fluid filled cylindrical shell: Application to Gelification process monitoring by surface acoustic wave. Loic Martinez, Brahim Senouci, Stephane Serfati (Laboratoire d'Electronique Applique de Cergy Pontoise, Universite de Cergy Pontoise, 5 mail Gay-Lussac, F 95031 Neuville sur Oise Cedex, France), Marcel Gindre, Jean-Yves Le Huerou (Laboratoire dImagerie Parametrique, 75270 Paris Cedex 06, France), and Pascal Griesmar (Laboratoire de Chimie des Materiaux Inorganiques, 95 031 Neuville sur Oise Cedex, France)

During gel formation, the material passes from the liquid state to the solidlike state. In order to characterize this liquid-to-solid transition, the low-frequency fluid-born plate mode A-wave is used. The A-wave has interesting properties to be nonattenuated-very dispersive at lowfrequency thickness products (fd), and nondispersive for high fd (the Scholte wave is the high fd limit). The axial propagation of the A-wave is studied along a glass cylindrical shell in the mid to high fd range $(0.5$ $\mathrm{MHz} \mathrm{mm}-1.5 \mathrm{MHz} \mathrm{mm}$ ). The A-wave is generated and received at one extremity of the tube by a contact broadband transducer excited by a pulse. In the calibration test, the cylindrical shell is filled with water and surrounded by air outside. The A-wave dispersion curve is extracted by short time Fourier transform; comparison with the theoretical dispersion curves is very good. For the gelification monitoring, the water is replaced by the gel in its liquid state. A-wave time signals are recorded during the gelification process. The evolution of the longitudinal speed during the gelification time is deduced from the A-wave dispersion curves.

\section{$8: 45$}

3aPA4. Differential surface acoustic wave IDT gyroscope. Jose K.A, Anshu Mehta, and Vijay K. Varadan (212 EES Bldg., Penn State Univ., University Park, PA 16802)

This paper presents the design and experimental evaluation of a gyroscope based on differential design of a surface acoustic wave (SAW) resonator and a sensor on a piezoelectric substrate. The resonator is divided into two halves, one with metallic dot arrays and other without any metallic dots. Standing waves are formed inside both cavities. The particles at the antinodes of the standing wave pattern experience large amplitude of vibration that serves as the reference vibrating motion for this gyroscope. The metallic dots strategically positioned at the antinode locations experience Coriolis force due to rotation and acoustically amplify the magnitude in the orthogonal direction. However, the other half of the resonator cannot generate any secondary SAW. A wideband SAW sensor arranged orthogonal to the SAW resonator picks up these two secondary SAWs and is fed to a differential amplifier. Any drift in the gyroscope signal can be completely eliminated by this novel design. The performance of this 74.2$\mathrm{MHz}$ gyroscope shows very high sensitivity and dynamic range, which is ideal for many commercial applications. Unlike other MEMS gyroscopes, this gyroscope has a planar configuration with no suspended resonating mechanical structures, thereby being inherently robust and shock resistant.
3aPA5. Diffraction correction for precision SAW velocity measurements. Alberto Ruiz M. and Peter B. Nagy (Dept. of Aerosp. Eng. and Eng. Mech., Univ. of Cincinnati, Cincinnati, OH 45221-0070, ruiza@email.uc.edu)

Surface acoustic wave (SAW) dispersion measurements can be used to nondestructively characterize shot-peened, laser shock-peened, and other surface-treated specimens. In recent years, there have been numerous efforts to separate the contribution of surface roughness from those of nearsurface material variations, such as residual stress, texture, and increased dislocation density. This talk addresses the problem that a perceivable dispersive effect, similar to the one found on rough shot-peened specimens, is exhibited by untreated smooth surfaces as well. The dispersion measurements were performed using laser-ultrasonic scanning combined with special digital signal-processing methods. The observed dispersion effect is on the order of $0.1 \%$, which is comparable to the expected velocity change produced by near-surface compressive residual stresses in metals below their yield strength. The cause of this apparent dispersion is the diffraction of the SAW as it travels over the surface of the specimen. It is demonstrated that a diffraction correction may be introduced to increase the accuracy of surface wave dispersion measurements. A simple diffraction correction model was developed for surface waves; this correction was subsequently validated by laser-interferometric surface wave velocity measurements on 2024-T351 aluminum specimens.

\section{9:15}

3aPA6. Thin film characterization using resonant ultrasound spectroscopy. J. R. Gladden, Jin H. So, Rajdeep Pradhan, and J. D. Maynard (Dept. of Phys., Penn State Univ., University Park, PA 16803, jgladden@psu.edu)

With mechanical and electrical components approaching nanoscale dimensions, there is great interest in the properties of thin films. Some properties, such as structure and phase transitions, can be probed with measurements of elastic constants. We have been using Resonant Ultrasound Spectroscopy (RUS) to probe such properties for a variety of thin film materials including colossal magnetoresistance (CMR) materials, carbon nanotubes, and a magnetic semiconducting film on GaAs. By accurately characterizing the substrate, the elastic constants and damping of a deposited film can be determined from changes in the frequency and quality factor $(Q)$ of the sample resonances. Issues of precision and accuracy in RUS measurements are important for application to thin films. If one is interested in relative changes in properties (e.g., resulting from temperature variations, phase transitions, etc.), the precision from sharp resonances $(Q>5,000)$ is more than adequate. However, since a film may occupy only $1 / 1000$ of the sample, attaining high accuracy is challenging, as uncertainty in sample dimensions and crystallographic orientation can have severe effects on results. Temperature data for the magnetic films mentioned and attenuation of carbon nanotube films will be presented. [Work supported by ONR.]

\section{9:30}

3aPA7. Effect of hydrochloric acid on sound absorption and relaxation frequency in magnesium sulfate solutions. F. H. Fisher (Marine Physical Lab., Scripps Inst. of Oceanogr., UCSD, La Jolla, CA 92093-0701)

The epic work of Kurtze and Tamm on sound absorption spectroscopy in divalent sulfate electrolyte solutions (1953) from the low-kHz region up to over $200 \mathrm{MHz}$ revealed astonishing variability at frequencies below 10 $\mathrm{MHz}$ and a common relaxation frequency at about $200 \mathrm{MHz}$. For magnesium sulfate [Epsom salts] solutions, the salt producing $30 \times$ the absorption of fresh water below the $100-\mathrm{kHz}$ region in the oceans at low concentrations $[\sim 0.02 \mathrm{moles} / \mathrm{liter}]$, Kurtze and Tamm investigated the effects of adding $\mathrm{HCl}$ or $\mathrm{H}_{2} \mathrm{SO}_{4}$. They found that as formal $p \mathrm{H}$ increased, the results were different for these acids in reducing the sound absorption. Fisher (1983) found that if the absorption was plotted against free hydrogen, ion concentration was the same. We used the 100-liter titanium sphere, a spare ballast tank from the WHOI submarine ALVIN. With precise 
temperature control, we found an increase in the relaxation frequency as HC1 was added in conjunction with the reduction in sound absorption. The results will be presented and an explanation will be proposed in the context of the Eigen and Tamm multistate dissociation model for $\mathrm{MgSO}_{4}$ (1962) which explains the effects of pressure on both absorption and conductance. [Work supported by ONR.] The author acknowledges C. C. Hsu for his work on this project.

\section{9:45}

3aPA8. Using resonance ultrasound spectroscopy to study colossal magnetoresistance in thin films. Rajdeep Pradhan, Jin H. So, J. R. Gladden, and J. D. Maynard (Dept. of Phys., Penn State Univ., University Park, PA 16803,rsp133@psu.edu)

Materials displaying colossal magnetoresistance (CMR) are candidates for important applications such as high density data storage. When the CMR material is in the form of a thin film on a substrate, the strain which results from lattice mismatch with the substrate alters the behavior of the material. As for CMR materials in bulk form, there should be a correlation between the CMR behavior and the structural and elastic properties of the strained thin films. Measurements of the elastic properties of thin films are obtained using a novel small sample resonant ultrasound spectroscopy (RUS) technique. Thin films of the magnetic material are deposited on a substrate and the difference in natural frequencies are then used to obtain the elastic constants of the film. The dominant CMR materials are perovskite manganites, a transition metal oxide. They exhibit a variety of exotic structural, magnetic and electrical behaviors, which is yet to be understood. In this paper measurements of the electrical resistance and elastic constants of 200 and $400 \mathrm{~nm}$ thin films, as well as unstrained samples, of the CMR material $\mathrm{La}(0.7) \mathrm{Ca}(0.3) \mathrm{MnO}_{3}$ are presented. [Work supported by ONR.]

WEDNESDAY MORNING, 5 JUNE 2002

GRAND BALLROOM 4, 8:15 TO 11:50 A.M.

\title{
Session 3aPP
}

\section{Psychological and Physiological Acoustics and Speech Communication: Honoring the Contributions of Ira J. Hirsh}

\author{
Janet M. Weisenberger, Cochair \\ College of Social and Behavioral Science, The Ohio State University, 1010 Derby Hall, 154 North Oval Mall, \\ Columbus, Ohio 43210 \\ Judith L. Lauter, Cochair \\ Department of Human Services, Stephen F. Austin University, P.O. Box 13019, SFA Station, Nacogdoches, Texas 75962
}

\section{Invited Papers}

8:15

3aPP1. Ira at 80: The acronyms of a career in acoustics. Janet M. Weisenberger (Speech and Hearing Sci., Ohio State Univ., Columbus, OH 43210, jan+@osu.edu)

In a career that spans some 54 years to date, the name of Ira J. Hirsh has been associated with significant scientific contributions to psychoacoustics, outstanding mentoring of research scientists, and dedicated service to the fields of acoustics, audiology, and psychology. It is a career that can be traced by acronyms that are part of the daily vocabulary of hearing scientists. These include acronyms of location: Early work at the Psychoacoustics Laboratory at Harvard (PAL), a long tenure in research at the Central Institute for the Deaf (CID), service as faculty member, chair, and dean at Washington University (WashU); acronyms of professional societies that have honored him: Acoustical Society of America (ASA), International Commission of Acoustics (ICA), American Psychological Association (APA), American Psychological Society (APS), American Speech-Language-Hearing Association (ASHA), American Association for the Advancement of Science (AAAS); acronyms of his service to the National Academy of Science: National Research Council (NRC), Commission on Behavioral and Social Science and Education (CBASSE); and acronyms of his contributions to psychoacoustics: Masking Level Difference (MLD), Temporal Order Judgments (TOJ). In large part, these acronyms are part of our vocabulary because of Ira's contributions, and tracing them over the past half-century yields a substantive look at the development of the field of hearing science.

\section{8:30}

3aPP2. Events, sequences, and patterns: Hirsh's prescient proposals. Charles S. Watson (Dept. of Speech and Hearing Sci., Indiana Univ., Bloomington, IN 47405, watson@indiana.edu)

Over four decades ago, Ira Hirsh was one of the first to recognize the need for auditory research to expand beyond the study of single tones, noise burst, and clicks. He wrote, "We propose to examine auditory perception at a more complex level. The discrimination among and identification of single sounds, which we shall refer to as acoustic events, is undoubtedly an important part of the perceptual process, but perhaps more important are the rules by which we distinguish and identify sequences of acoustic events. Auditory psychophysics has been concerned with the acoustic characteristics of the sequence parts, the events; but we need to know more about the ways in which the parts combine to form patterns which, since they are generated in time, we may call sequences" [J. 
Acoust. Soc. Am. 31 (1959)]. This must be one of the more prescient proposals of that era, followed as it was not only by Hirsh's series of studies of temporal processing, but by a raft of other adventures into the world he forecast, including research on a great variety of auditory patterns and sequences. Theoretical interpretations of that work and of the general problem areas that Hirsh pioneered will be discussed.

8:50

3aPP3. It's about time: Presentation in honor of Ira Hirsh. Ken Grant (Walter Reed Army Medical Ctr., Army Audiol. and Speech Ctr., Washington, DC 20307-5001)

Over his long and illustrious career, Ira Hirsh has returned time and time again to his interest in the temporal aspects of pattern perception. Although Hirsh has studied and published articles and books pertaining to many aspects of the auditory system, such as sound conduction in the ear, cochlear mechanics, masking, auditory localization, psychoacoustic behavior in animals, speech perception, medical and audiological applications, coupling between psychophysics and physiology, and ecological acoustics, it is his work on auditory timing of simple and complex rhythmic patterns, the backbone of speech and music, that are at the heart of his more recent work. Here, we will focus on several aspects of temporal processing of simple and complex signals, both within and across sensory systems. Data will be reviewed on temporal order judgments of simple tones, and simultaneity judgments and intelligibility of unimodal and bimodal complex stimuli where stimulus components are presented either synchronously or asynchronously. Differences in the symmetry and shape of "temporal windows" derived from these data sets will be highlighted.

9:10

3aPP4. The times of Ira Hirsh. Pierre L. Divenyi (Speech and Hearing Res., VA Medical Ctr., Martinez, CA 94553)

Ira Hirsh was among the first to recognize that the auditory system does not deal with temporal information in a unitary way across the continuum of time intervals involved in speech processing. He identified the "short" range (extending from 1 to $20 \mathrm{~ms}$ ) as that of phase perception, the "medium" range between 20 and $100 \mathrm{~ms}$ as that in which auditory patterns emerge, and the "long" range from $100 \mathrm{~ms}$ on as that of separate auditory events. Further, he was also among the first to recognize that auditory time perception heavily depended on the spectral context. A study of the perception of sequences representing different temporal orders of three tones, by Ira and the author [e.g., Divenyi and Hirsh, J. Acoust. Soc. Am. 64, 1369-1385 (1978)] demonstrated the dependence of auditory sequence perception on both time range and spectral context, and provided a bridge between Hirsh's view of auditory time and Bregman's view of stream segregation [Auditory Scene Analysis (MIT Press, Cambridge, MA, 1991)]. A subsequent search by the author for psychophysical underpinnings of the cocktail-party phenomenon [e.g., Divenyi et al., Moh. Mtn. Workshop Appl. SPAA (IEEE, Bellingham, WA, 1997)] suggest that the segregation of simultaneous streams of speech may rely on the ability to follow spectral changes in the demisyllabic-to-syllabic (100- to 200-ms) range, i.e., Ira's "long" range.

\section{9:30}

3aPP5. Ira as a pioneer in audiology: His contributions to the clinical measurement of hearing and hearing impairment. C. Formby (Univ. of Maryland, Baltimore, MD 21201) and J. P. Gagne (Univ. de Montreal, Montreal, QC H3C 3J7, Canada)

Ira Hirsh's contributions to clinical science and research are diverse and significant. In fact, approximately one-third of the 100+ publications that Ira lists in his curriculum vitae (CV) are clinical in nature, dealing with various aspects of audiology, deafness, hearing aids, aural rehabilitation, and speech and language pathology. The majority of these citations, fully one-quarter of his publication list, addresses problems specific to the clinical measurement of hearing and hearing impairment. Undoubtedly, the most influential of these published works appears in his CV under the citation "The Measurement of Hearing." The forward for this publication, his only textbook, was penned in June, 1952 (now precisely half a century past at the time of this session). The aims of this presentation are to (1) provide perspective on the fundamental importance of his virtually timeless text in shaping the fledgling discipline of audiology, and (2) celebrate Ira's many contributions to the profession and practice of audiology. [Preparation for this presentation was supported, in part, by a K24 career development award from NIDCD.]

\section{9:50-10:10 Break}

\section{0:10}

3aPP6. Ira Hirsh and oral deaf education: The role of audition in language development. Ann Geers (Central Inst. for the Deaf, 4560 Clayton Ave., St. Louis, MO 63110)

Prior to the 1960s, the teaching of speech to deaf children consisted primarily of instruction in lip reading and tactile perception accompanied by imitative exercises in speech sound production. Hirsh came to Central Institute for the Deaf with an interest in discovering the auditory capabilities of normal-hearing listeners. This interest led him to speculate that more normal speech development could be encouraged in deaf children by maximizing use of their limited residual hearing. Following the tradition of Max Goldstein, Edith Whetnall, and Dennis Fry, Hirsh gave scientific validity to the use of amplified speech as the primary avenue to oral language development in prelingually deaf children. This "auditory approach," combined with an emphasis on early intervention, formed the basis for auditory-oral education as we know it today. This presentation will examine how the speech perception, language, and reading skills of prelingually deaf children have changed as a result of improvements in auditory technology that have occurred over the past 30 years. Current data from children using cochlear implants will be compared with data collected earlier from children with profound hearing loss who used hearing aids. [Work supported by NIH.] 
No session honoring Ira Hirsh would be complete without a personal reflection of his enormous influence on his colleagues, students, and friends. During his career in St. Louis that covered the entire second half of the 20th Century (and still continues), Ira interacted with numerous colleagues at Central Institute for the Deaf, where he served as a senior scientist, Director of Research, and Institute Director. His second parallel career was at Washington University, where he was Professor of Psychology, and included a term as Dean of the Faculty of Arts and Sciences. This tribute will provide reflections and reminiscences of what it was like to work with, and learn from, Ira Hirsh.

10:50

3aPP8. Parameter space of the dichotic speech experiment. Robert C. Bilger (Dept. of Speech \& Hearing Sci., Univ. of Illinois at Urbana-Champaign, 901 S. 6th St., Champaign, IL 61820, r-bilger@uiuc.edu) and Charles E. Speaks (Univ. of Minnesota, Minneapolis, MN 55455)

The experiment in which different CV nonsense syllables are presented simultaneously to each ear and the listener is asked to identify both consonants often results in more correct responses to syllables presented to the right than to the left ear, the so-called right-ear advantage (REA). The REA is generally interpreted as an indication that the left hemisphere processes speech more efficiently than does the right hemisphere. As the experiment is usually conducted, however, it has the parameter space of 3 (i.e., requires three numbers to completely specify the results). Using data obtained independently in each of our labs, it will be shown that the three parameters necessary to uniquely specify the results of the experiment are the probability that the (1) first response is correct; (2) first response is to the syllable presented to the right ear; and (3) second response will be incorrect given that the first response was correct. The ear advantage $(\mathrm{R}, 0$, or $\mathrm{L})$ is predicted to the nearest percent using the first two parameters; but, as of this moment, the exact fourfold matrix for a listener cannot be predicted.

11:10

3aPP9. Transcending boundaries with Ira Hirsh. Punita G. Singh (Sound Sense, C6-6046 Vasant Kunj, New Delhi 110070, India, soundsense@vsnl.com)

Ira Hirsh has made many contributions to various fields of acoustics from speech, hearing, psychological and physiological acoustics, to musical and architectural acoustics. It was a privilege for me to have been his student in all these areas, and to have had him as a guide through masters and doctoral degree programs that focused on topics that lie at the boundaries connecting these disciplines. Ira was not a prescriptive advisor, imposing particular research topics or procedures on his graduate students. Rather, he encouraged originality, innovation, and personal goal setting. He would subtly suggest starting points and provide landmarks as references, rather than explicit directions leading to them. One had to navigate the path by ones own wits. This approach encouraged lateral, out-of-the box thinking, while also leading to respectful appreciation of historic trajectories in scientific research. During our time together, we worked on several aspects of music, including, rhythm, melody, pitch, and timber perception. Some of this work will be recapitulated, highlighting Ira's role in its exposition and development. His multidimensional personality, astute insights, colorful remarks, wry humor, care, and concern are qualities to be cherished—beyond the boundaries of campus, city, country, and contemporaneity.

\section{$11: 30$}

3aPP10. Ad cerebrum per scientia: Ira Hirsh, psychoacoustics, and new approaches to understanding the human brain. Judith Lauter (Human Neurosci. Lab., Dept. of Human Services, Stephen F. Austin State Univ., Box 13019, SFA Station, Nacogdoches, TX 75962)

As Research Director of CID, Ira emphasized the importance of combining information from biology with rigorous studies of behavior, such as psychophysics, to better understand how the brain and body accomplish the goals of everyday life. In line with this philosophy, my doctoral dissertation sought to explain brain functional asymmetries (studied with dichotic listening) in terms of the physical dimensions of a library of test sounds designed to represent a speech-music continuum. Results highlighted individual differences plus similarities in terms of patterns of relative ear advantages, suggesting an organizational basis for brain asymmetries depending on physical dimensions of stimulus and gesture with analogs in auditory, visual, somatosensory, and motor systems. My subsequent work has employed a number of noninvasive methods (OAEs, EPs, qEEG, PET, MRI) to explore the neurobiological bases of individual differences in general and functional asymmetries in particular. This research has led to (1) the AXS test battery for assessing the neurobiology of human sensory-motor function; (2) the handshaking model of brain function, describing dynamic relations along all three body/brain axes; (3) the four-domain EPIC model of functional asymmetries; and (4) the trimodal brain, a new model of individual differences based on psychoimmunoneuroendocrinology. 


\title{
Session 3aSAa
}

\section{Structural Acoustics and Vibration: Scattering; Composite Materials}

\author{
Joseph W. Dickey, Chair \\ Center for Nondestructive Evaluation, The Johns Hopkins University, 3400 North Charles Street, Baltimore, Maryland 21218
}

Contributed Papers

\section{8:00}

3aSAa1. Chaos, catastrophe, and critical frequency points on elastic shells. Michael Werby (NRL, Code 7181, Stennis Space Center, MS 39529) and H. Uberall (Dept. of Phys., Catholic Univ. of America, Washington, DC)

Exact equations exist for the determination of the backscattered signals excited by acoustic signals on submerged elastic shells. In the absence of resonances these signals may be cast in terms of conservation principles of inertial components of the interaction of the signal with the object. This indeed leads to an adequate description of the acoustic background of such targets. What is determined to be critical is the inclusion of an entrained mass related to the displacement of the mass equivalent of the fluid due to the movement of the object. But what happens to such inertial effects in the presence of elastic resonances? We explore this issue and demonstrate that the effective or inertial component ranges from zero to infinity over a small frequency range that characterizes the resonance width. Over this range there is also a phase shift of 1800 . We may as well examine the effect of radiation loading in this range. It is also possible to investigate a phase plot of the real and imaginary components of the effective mass factor which leads to closed trajectories for certain classes of resonances and to hyperbolic trajectories for other types. The meaning of these observations is discussed. [Work supported by NRL and ONR.]

\section{$8: 15$}

3aSAa2. Backscattered acoustic signal from submerged water filled elastic shells can be decomposed into body resonances and eigenmodes of the contained water. Michael Werby (NRL Code 7180, Stennis Space Center, MS 39529) and H. Uberall (Dept. of Phys., The Catholic Univ. of America, Washington, DC 20064)

Acoustic signals scatter from an elastic shell and excite elastic shell resonances. For evacuated shells resonances due to proper Lamb waves, $\mathrm{A} 0$ and S0 waves and the pseudo-Stoneley resonances, are the only ones allowed. When the shell is fluid filled then the eigenfrequencies of the included fluid may be excited. Further, the presence of the fluid may alter the elastic body resonances. When the impedance of the entrained fluid is not much smaller than the mechanical impedances of the elastic material then the scattered signal is greatly influenced by the entrained fluid, but only at their allowed eigenfrequencies. Since the fluid is entrained this leads to a discrete spectrum and an eigenvalue problem which is quite manageable. In this work we outline a method for determining the eigenfrequencies as well as their nature and use their values to isolate the actual body resonances due to the elastic material. We also illustrate the influence of the presence of the entrained fluid on the elastic resonance frequencies. [Work supported by NRL and ONR.]
8:30

3aSAa3. Visualization of the energy flow for a guided forward wave in and around a fluid loaded elastic cylindrical shell: Color coding of the Poynting vector field. Cleon E. Dean (Phys. Dept., P.O. Box 8031, Georgia Southern Univ., Statesboro, GA 30460-8031) and James P. Braselton (Dept. of Mathematics and Computer Sci., Georgia Southern Univ., P.O. Box 8093, Statesboro, GA 30460-8093)

Previous work [Cleon E. Dean and James P. Braselton, "Visualization of the energy flow for a guided forward wave in and around a fluid loaded elastic cylindrical shell," J. Acoust. Soc. Am. 109, 2379 (2001)] showed the energy flow for a forward propagating fluid loaded elastic cylindrical shell at the resonance frequency. The results were difficult to interpret since although the two counterpropagating guided waves were separated and displayed independently, the conventional grid of vector arrows was hard to interpret in part because they were hard to see individually. The current work uses color encoding to show the Poynting vector field. Hue is used to indicate the direction of the energy flow while intensity of the color is used to denote the magnitude of the Poynting vector at that point.

\section{$8: 45$}

3aSAa4. Investigation of the sound transmission behavior of a chamber core cylinder. Deyu Li and Jeffrey S. Vipperman (Dept. of Mech. Eng., Univ. of Pittsburgh, 531 Benedum Hall, Pittsburgh, PA 15228)

Several kinds of novel composite structures, such as advanced grid stiffened (AGS) and chamber core (CC) structures have been designed, fabricated, and investigated for both civil and military applications. The chamber core composite is a novel advanced sandwich-type structure that is created by filament winding an inner shell onto a cylindrical mandrel, arranging previously fabricated U-shaped channels around the perimeter of this shell to form the inner chamber walls, and filament winding an outer shell followed by a co-cure process. In this study, the structural/ acoustic behavior of a normal composite chamber core cylinder is investigated both theoretically and experimentally. Lightly coupled structural and acoustic modal parameters are identified using experimental modal analysis techniques. The properties of sound transmission loss (TL) of the cylinder are also investigated experimentally. The effect of the structural/ acoustic natural frequencies and the damping on the sound transmission loss is analyzed. Finally, passive control strategies are discussed, and several passive control materials for improving the sound transmission loss $(0-500 \mathrm{~Hz})$ of the cylinder are experimentally evaluated. [Work sponsored by the Air Force Research Laboratory Space Vehicles Directorate (AFRL/ VS), POC Dr. Steven Lane, (505) 846-9944.]

\section{9:00}

3aSAa5. Sound absorption characteristics of cenosphere enriched cement and asphalt concrete. Vikrant Tiwari, Arun Shukla (Dynamic Photomechanics Lab., Dept. of Mech. Eng. and Appl. Mech., Univ. of Rhode Island, Kingston, RI 02881), and Arjit Bose (Univ. of Rhode Island, Kingston, RI 02881)

A detailed experimental study has been conducted to determine the effect of addition of cenospheres on the acoustic properties of cement matrix and asphalt concrete. Cenospheres are hollow ceramic microballoons, being a waste product of thermal power plants, they are relatively 
inexpensive and their use has the added benefit of decreasing the strain on the environment. Specimens were made with a different volume fraction of cenospheres $(0 \%$ to $70 \%$ in increments of 10$)$ to observe the change in acoustic properties with an increase in the cenosphere content. Specimens of different sizes were tested to study the acoustic characteristics of the materials for a wide range $(125$ to $4000 \mathrm{~Hz})$ of frequencies. The effect of an increase in the cenosphere content on the dilatational wave velocity in the specimens was also studied with the help of acoustic transducers. It was noted that, although the wave velocity remains relatively unaffected with a change in the cenosphere content, the addition of cenospheres improves the sound absorption characteristics of the cement matrix and asphalt concrete with a considerable decrease (up to $41 \%$ ) in the density of the material. It has also been noted that the sound absorption of cement increases with the addition of cenosphere up to $40 \%$ volume fraction and starts decreasing with the further addition of cenospheres.

\section{9:15-9:30 Break}

\section{9:30}

3aSAa6. An equivalent solid $(u)$ formulation for poroelastic materials. Dominic Pilon and Raymond Panneton (GAUS, Dept. of Mech. Eng., Univ. de Sherbrooke, 2500 boul. de l'Universite, Sherbrooke QC J1K 2R1, Canada)

Finite element formulations based on Biot's poroelasticity equations have been used extensively throughout recent years. The most common are Biot's displacement $(\underline{u}, \underline{U})$ and mixed displacement-pressure $(\underline{u}, p)$ formulations. They are used to predict the structural and acoustical behavior of poroelastic mediums within multilayered structures. These models, while accurate, lead to the resolution of large linear systems: they need, respectively, six and four degrees of freedom per node in order to efficiently describe the poroelastic medium's vibroacoustic behavior in 3-D problems. In this paper, a simplified displacement $(\underline{u})$ formulation is presented. It is also based on Biot's equations, but requires only three degrees of freedom per node, related to the solid phase displacement field, to describe the behavior of poroelastic mediums in 3-D problems. The development of the governing $(\underline{u})$ equations for the equivalent solid poroelastic formulation is detailed. The limitations of this approach are also discussed. Numerical and experimental validations are presented in order to show the accuracy and effectiveness of the formulation within its prescribed field of application.

\section{0:00}

3aSAa7. Genetic encoding for sound package configuration optimization. Heng-Yi Lai (Dept. of System and Network, United Technologies Res. Ctr., 411 Silver Ln., MS129-17, East Hartford, CT 06108, laih@utrc.utc.com)

The acoustic properties of poroelastic materials can be accurately predicted by the Biot-Allard model if the required acoustic parameters have been successfully characterized. In conjunction with the transfer matrix approach, one can use the model to analytically design an acoustic liner comprising different materials that can provide better performance or cost reduction advantages over their homogeneous counterparts. However, optimizing the layer configuration remains to a challenging task as it is a combinatorial problem and the search for the optimal configuration can be difficult or expensive if simply based on the build-and-test approach. The
Generic Algorithm (GA) was identified as a better optimization method over the gradient-based methods for this task due to its nature in finding the combinatorial solutions. This approach is, to the best of our knowledge, novel in applying the GA to this design problem. The performance of the GA method is compared with the exhaustive search in maximizing the sound absorption coefficient of an acoustic liner with constraints set for its cost, thickness, and total weight. The features of the Generic Algorithm and the preliminary proof-of-concept results will be discussed and presented.

\section{0:15}

3aSAa8. On the dynamics of fuzzy structures. Jean-Mathieu Mencik and Alain Berry (GAUS, Mech. Eng. Dept., Univ. of Sherbrooke, Sherbrooke, QC J1K 2R1, Canada, Jean-Mathieu.Mencik@gaus.gme.usherb.ca)

In this presentation, one proposes a prediction at low- and midfrequencies of the dynamics of fuzzy structures. As introduced by Soize, the term "fuzzy structure" designates a master structure, whose geometrical, material characteristics, boundary conditions, and excitations are known, coupled with complex systems, called the structural fuzzy or fuzzy, whose characteristics are imprecisely known. Previous works done on this subject analyze the concept of a master structure coupled with a locally homogeneous fuzzy, composed of a large number of linear oscillators excited by their supports. In the present work, the concept of a homogeneous fuzzy is extended to an elastic continuum medium. One theoretically formulates the action of the elastic fuzzy on the master structure: it is shown that the proposed formulation is different from the solution proposed by Soize, derived from the model of a linear oscillator excited by its support. The proposed theory is successfully applied to the case of a homogeneous structural fuzzy composed of a large number of elastic bars whose lengths and cross sections are randomly chosen.

$10: 30$

3aSAa9. Dissipation induced by substructures and distribution of vibratory energy in a complex system. Sunghoon Choi and Yang-Hann Kim (Dept. of Mech. Eng., KAIST, Science Town, Taejon 305-701, Republic of Korea)

This work is concerned with the problem of energy transfer that takes place between a master structure and the substructures attached to it. The response of the system is characterized by the impedance of the substructures and it determines whether the induced damping is real or apparent. When the dissipation is real the master structure has a larger loss factor than that of the substructures and there will be continuous transfer of vibratory energy from the master structure to substructures. However, in the case of apparent damping, one can observe that the vibration energy is transferred back and forth between the master and substructures. Some combinations of the master and substructures have been considered to examine this phenomenon and to determine the criteria for damping. It has found that a modal overlapping condition, which corresponds to bandwidths that exceed the spacing of those natural frequencies, is crucial in determining the characteristics of the system damping. The result of this paper is consistent with that found with the fuzzy structure and SEA framework. [Work sponsored by Ministry of Education, Korean Government under the BK21 program and Ministry of Science and Tech., Korean Government under National Research Lab. program.] 


\title{
Session 3aSAb
}

\section{Structural Acoustics and Vibration and Archives and History: History of Structural Acoustics and Vibration}

\author{
Joseph W. Dickey, Chair \\ Center for Nondestructive Evaluation, The Johns Hopkins University, 3400 North Charles Street, Baltimore, Maryland 21218
}

\section{Invited Paper}

\section{1:00}

\begin{abstract}
3aSAb1. History of structural acoustics and vibrations in the Acoustical Society of America. David Feit, Murray Strasberg (Carderock Div., Naval Surface Warfare Ctr., 9500 MacArthur Blvd., West Bethesda, MD 20817), and Eric E. Ungar (Acentech, Inc., Cambridge, MA 02138-1118)

Structural acoustics refers to the interaction of sound and structures- the response of structures to sound, the radiation of sound from vibrating structures, and the effect of the acoustic medium on the structural vibrations. Interest in these subjects increased greatly during the 1930s and 40s because of practical applications in the design of microphones and loud speakers used in telephones, radios, and electronic phonographs. The combination of electrical and mechanical systems lead to the use of electrical engineering concepts such as impedance, circuits, and electrical analogies, in the analysis of mechanical systems. In later years, much of the work dealt with various aspects of underwater structures, prompted by U.S. Navy interests. The field, which began with classical analytical mechanics applications, has progressed to new approaches, including statistical energy analysis, near-field acoustical holography, fuzzy structures, active control of vibrations, and smart materials. In recognition of these new developments, the name of the technical committee was changed in 1987 from "Shock and Vibration" to "Structural Acoustics and Vibration."
\end{abstract}

WEDNESDAY MORNING, 5 JUNE 2002

GRAND BALLROOM 3, 7:55 TO 9:45 A.M.

\section{Session 3aUWa}

\section{Underwater Acoustics: Array Performance}

\author{
Peter Gerstoft, Chair \\ Marine Physics Laboratory, Scripps Institution of Oceanography, University of California, San Diego, 8820 Shellback Way,
} La Jolla, California 92093-0238

Chair's Introduction-7:55

Contributed Papers

8:00

3aUWa1. Acoustic tracking of towed-array hydrophone positions during tow-vessel maneuvers. Alec J. Duncan (Ctr. for Marine Sci. and Technol., Curtin Univ., GPO Box U1987, Perth, WA 6845, Australia, a.duncan@cmst.curtin.edu.au), Darryl McMahon (Defence Sci. and Technol. Organisation, HMAS Stirling, Garden Island, Western Australia), and Alessandro Ghiotto (Nautronix Ltd., 108 Marine Terrace, Fremantle, WA 6160, Australia)

This paper describes an experiment in which a vessel towing an array of hydrophones executed a series of maneuvers designed to bring the array into orientations favorable for imaging noise sources on the tow-vessel. Signals from tracking beacons on the tow-vessel and transient signals from implosive sources deployed from a second vessel were received on the array hydrophones and were used to obtain independent estimates of hydrophone position. Array shape estimation and tracking algorithms are described and the results of applying these algorithms to the experimental data are presented.
$8: 15$

3aUWa2. Adaptive beamforming of a towed array during a turn. Peter Gerstoft, William S. Hodgkiss, W. A. Kuperman, Heechun Song (Marine Physical Lab., Scripps Inst. of Oceanogr., La Jolla, CA 92093-0238), Martin Siderius, and Peter L. Nielsen (SACLANT Undersea Res. Ctr., 19138 La Spezia, Italy)

During maneuvering, towed-array beamforming will degrade if a straight array is assumed. This is especially true for high-resolution adaptive beamforming. This problem can be reduced if a proper curved array is assumed. The tow vessel's Global Positioning System (GPS) can be used to estimate this curvature, and this reduces the need for instrumentation in the array. Based on estimated array shape from GPS, both the conventional beamformer and the white-noise-constrained (WNC) adaptive beamformer are shown to track the source well. For calculating the steering vector in the WNC approach, a matrix inversion of the cross-spectral density matrix is involved. This matrix inversion can be stabilized by averaging the cross-spectral density matrix over neighboring frequencies. 
3aUWa3. Subarray partitions of large aperture planar towed arrays. Jennifer A. Watson (Lincoln Lab., MIT, 244 Wood St., Lexington, MA 02420, jwatson@LL.mit.edu), Arthur B. Baggeroer (MIT, Cambridge, MA 02139), Lisa M. Zurk, and Brian H. Tracey (Lincoln Lab., MIT, Lexington, MA 02420)

The current focus of passive detection and localization is in littoral regions where acoustic propagation becomes complicated by severe bottom interaction. The resultant high-transmission loss motivates the need for high-array gain for effective performance over long ranges. Large planar seismic arrays, with aperture dimensions upwards of $3 \mathrm{~km} \times 0.5 \mathrm{~km}$, have potential to achieve high gain and good resolution when using matched field processing. In realistic environments, however, large arrays are suceptible to signal gain degradation mechanisms, particularly due to spatial decorrelation of the signal and non-stationary environments. One approach to overcoming this is partitioning the array. Subarray processing reduces stationarity requirements and extracts optimum coherent gain, thus achieving higher gain than that of smaller arrays. This work examines criteria for partitioning planar arrays to perform localization using MFP. Trade-offs between spatial resolution, array gain, and resilience to motion will be quantified and discussed. Performance of different subarray geometries will be presented using adaptive and conventional MFP. [Work sponsored by DARPA under Air Force Contract No. F1962800-00-C0002. Opinions, interpretations, conclusions, and recommendations are those of the authors and are not necessarily endorsed by the Department of Defense. AB's work was supported by ONR through the SECNAV CNO Chair.]

\section{$8: 45$}

3aUWa4. Passive localization algorithms. Edmund J. Sullivan 46 Lawton Brook Ln., Portsmouth, RI 02871)

An acoustic source can be localized by exploiting the fact that, if the range is not too great, the wavefront of the incoming acoustic energy can be assumed to be circular. This is sometimes called "Wavefront Curvature Ranging." Here, the estimation of range reduces to the estimation of the radius of a circle. In fact, if sufficient horizontal and vertical aperture is available, range, bearing and elevation can be estimated, given a minimum of four receivers. The technique is based on the measurement of the relative phases between pairs of receivers. In this paper, two methods of improving the performance of such localizers are investigated. In the case where the receivers are mounted on a moving platform, it is shown that exploitation of this motion can improve performance by utilizing information that is usually ignored in phase measurements. Also, since the motion introduces a time dependence in the true values of the time delays, a limit on the accuracy of their measurement is incurred. A recursive estimation process is proposed as a remedy for this problem. These techniques will be demonstrated by using simulated data.
3aUWa5. Beam intensity striations and applications. T. C. Yang (Naval Res. Lab., Washington, DC 20375)

Modal interferences, or striations, are analyzed and modeled for the beam outputs of a horizontal line array obtained by conventional beamforming. The enhancement of signal levels by the array gain (over that of a single element) makes the measurement of the invariant parameter more reliable even in the weak signal cases. It is shown that the signal beams exhibit the same striation pattern as that predicted (theoretically) for a single element. Specifically, for a broadside signal, the beam striation is identical to that of the single element plus a constant signal gain. For a non-broadside target, the signal beam intensity will be modified by a frequency-bearing dependent signal gain due to the signal spread over multiple beams (the multipath effect). Nonetheless the signal beam intensity still retains the same striation pattern (slope) as for a single element. At non-signal look directions, the sidelobe beams (those outside the canonical cones containing the signal arrivals) exhibit an entirely different striation pattern. The difference of signal striations between a fast rangerate, close range signal and distant, slow range-rate interferences can be used to suppress the interferences in array processing. [Work supported by ONR.]

\section{9:15}

3aUWa6. Preliminary results of horizontal array coherence from the 2001 ASIAEX South China Sea experiment. Theodore H. Schroeder, James F. Lynch, and Arthur Newhall (Woods Hole Oceanogr. Inst., MS\#11, Woods Hole, MA 02543, tschroeder@whoi.edu)

The 2001 ASIAEX (Asian Seas International Acoustics Experiment) South China Sea experiment was performed in April-May, 2001 off the southern coast of the People's Republic of China. This experiment was a combined acoustics/physical oceanography/marine geology effort that endeavored to measure basic acoustic propagation and scattering processes in a well-supported manner. As part of the experiment, a combination vertical/horizontal autonomous receiving array was deployed in $124 \mathrm{~m}$ of water, where it listened to a combination of moored and towed acoustic sources spanning a bandwidth of 50-600 Hz. These acoustic measurements were supported by geological surveys and extensive oceanographic measurements from fixed and towed instruments, providing a 3-D picture of the overall oceanography in the experimental area. The initial interest in this data centers about horizontal array coherence issues, as few experiments have had a horizontal array coupled with extensive environmental support, allowing for a direct correlation of the array coherence with the environment. The frequency dependence, time variability, and along-shelf vs. across-shelf variation of coherence are of particular interest. The latest results in these topics will be shown. [Work supported by ONR.]

\section{9:30}

3aUWa7. Effect of a ship's body on the beam pattern of its hydroacoustic array. Tran H. Dat and V. T. Grinchenko (Inst. of Hydromech., Natl. Acad. of Sci. of Ukraine, Zhelyabov Str. 8/4, Kiev 03035, Ukraine) 


\title{
Session 3aUWb
}

\section{Underwater Acoustics: Shallow Water Propagation and Signal Processing}

\author{
David M. Deveau, Chair \\ PSC 1012, Box 701, FPO, AA 34058-9998 \\ Chair's Introduction-10:10 \\ Contributed Papers
}

10:15

3aUWb1. Shallow water impact acoustics. David M. Deveau (Naval Undersea Warfare Ctr. Det. AUTEC, 801 Clematis St., West Palm Beach, FL 33401)

During a series of impact detection and tracking accuracy tests near Wallops Island, Maryland, an analysis was conducted on the acoustic impacts themselves. Since the impact area was very shallow (60 ft), the acoustic signature traveled unscathed between the 1000 yard baseline sensors. The initial goal was to implement deep water impact tracking algorithms in the shallow environment. Initially the in-water tracking system computed positions that were close to those which were laser sighted, but not close enough. Since the transient acoustic signature was very evident, hence a stable timing mark, an investigation into the acoustics surrounding the impact was undertaken. A review of the data showed small transients which occurred distinctly before the large transient detection that had routinely been considered as the impact itself. Using a threshold detection scheme, a relative set of timing marks were generated based on these early transients and were found to produce a more accurate placement.

\section{0:30}

3aUWb2. Channel characterization for communications in very shallow water. Robert J. McDonald, Kerry W. Commander, John S. Stroud, Jo Ellen Wilbur (Coastal Systems Station, 6703 W. Hwy. 98, Panama City, FL 32407-7001, commanderkw@ncsc.navy.mil), and Grant B. Deane (Univ. of California, San Diego, La Jolla, CA 92093-0238)

Broadband acoustic transmissions $(7 \mathrm{kHz}$ to $17 \mathrm{kHz})$ taken from July 4, 2000 to July 8, 2000, in the shallow water near Scripps Pier at La Jolla, California, are used to extract the time-varying channel parameters of coherence time and multipath time delay spread, as functions of frequency and the environmental variables of wave height and tidal fluctuations. Tidal fluctuations, which have a significant effect on water depth at the receiver and transmitter, are shown to strongly correlate to variations in the multipath delay spread. Variations in the coherence time for the channel, as measured by the drop off in correlation between initial and successive impulse responses, are shown to be inversely related to variations in the measured wave height. The coherence time of the channel was found to decrease with increasing center frequency. A scatter plot of the receiver signal-to-noise ratio as a function of wave height and water depth indicates when the shallow water environment allows a viable communications channel to exist.

\section{0:45}

3aUWb3. Estimating the velocity of a moving object submerged in an ocean waveguide with active sonar. Yi-san Lai and Nicholas Makris (MIT, 77 Masschusetts Ave., Cambridge, MA 02139)

Standard active sonar and radar systems are often used to estimate the velocity of a moving target in free space by resolving the Doppler shift of the scattered waveform. In an ocean waveguide, multimodal propagation and dispersion make the Doppler effects far more complicated than in free space. In a waveguide, multiple frequency components are typically present in the field scattered from a moving object even if the active source of radiation is harmonic. Applying a free space Doppler correction to the field scattered from a moving object in a waveguide is insufficient to account for the Doppler distortion of the signal in typical ocean waveguides and active sonar scenarios. Spectral and modal formulations for the Doppler-shifted field scattered from a horizontally moving target in a horizontally stratified medium have been developed by Lai and Makris [J. Acoust. Soc. Am. 110, 2724 (2001)]. These formulations are used with the maximum likelihood method to estimate the velocity of the moving target by modeling the multiply Doppler distorted scattered signal measured with noise in a shallow water waveguide. The performance of the maximum likelihood estimate is evaluated by asymptotic analysis of its bias and mean-square error as a function of signal-to-noise ratio.

\section{1:00}

3aUWb4. Time-frequency analysis and conditional moments of shallow-water sound propagation. Patrick J. Loughlin (Dept. of Elec. Eng., Univ. of Pittsburgh, Pittsburgh, PA 15261)

Shallow-water sound propagation is inherently nonstationary owing to such effects as geometric dispersion. The spectrogram has been a principal means to study the nonstationarities and dispersion characteristics of shallow-water sound propagation. In this talk, we give the low-order conditional spectral and wave number moments of sound propagation in dispersive environments, and show how they characterize the nonstationarities induced by channel dispersion on the propagating wave. [Work supported by ONR (N00014-02-1-0084).]

\section{1:15}

3aUWb5. Multipath in synthetic aperture sonar. John E. Piper (Coastal Systems Station, 6703 W. Hwy. 98, Panama City, FL 32407)

Synthetic aperture sonar is capable of producing high resolution images. As the detection range increases multipath effects can become important. In this paper we present the results of at-sea tests in the Gulf of Mexico and St. Andrew Bay with a dual-frequency $(20 \mathrm{kHz}$ and $180 \mathrm{kHz})$ synthetic aperture sonar. Surprisingly, it was found that range to water depth ratios as small as 4 often produced multipath signals significantly stronger than direct path signals. These effects on the synthetic aperture processing and image quality are discussed. 


\title{
Session 3pAO
}

\section{Acoustical Oceanography: Acoustical Oceanography Prize Lecture}

\author{
Peter F. Worcester, Chair \\ Scripps Institution of Oceanography, University of California, San Diego, 9500 Gilman Drive, \\ La Jolla, California 92093-0225
}

Chair's Introduction-12:55

Invited Paper

1:00

3pAO1. Ocean tomography, inverse methods, and broadband ocean acoustics. Bruce D. Cornuelle (Scripps Inst. of Oceanogr., UCSD, La Jolla, CA 92093)

Ocean acoustic tomography, as proposed by Munk and Wunsch in 1979, and as implemented by the Ocean Tomography Group, uses ray travel times to estimate ocean sound speed and currents. Earlier work by Medwin (1970) and Hamilton (1977) used pulse travel times as measures of integrated sound speed along paths at short and long range, respectively. Munk and Wunsch (1979) recognized that broadband transmissions between many instruments could be used with inverse methods [Backus and Gilbert (1967); Liebelt (1967)] to reconstruct 3D ocean sound speed fields from the travel times along multiple paths. Inverse methods are widely used in ocean acoustics and in physical oceanography, and the modern challenge is to incorporate time dependence into the inverse methods to take advantage of the improving ocean dynamical models. In addition, the understanding of broadband acoustic propagation has improved to the point of refining the sensitivity kernel for travel time measurements beyond the simple geometrical optics of ray paths. This paper will review the evolving use of forward and inverse methods in acoustical oceanography, primarily with application to acoustic tomography.

WEDNESDAY AFTERNOON, 5 JUNE 2002

GRAND BALLROOM 2, 2:00 TO 3:05 P.M.

\section{Session 3pID}

\author{
Interdisciplinary: Hot Topics in Acoustics \\ Mardi C. Hastings, Chair \\ Biomedical Engineering Center, The Ohio State University, 1080 Carmack Road, Columbus, Ohio 43210
}

Chair's Introduction-2:00

Invited Papers

2:05

3pID1. Hot topics: Signal processing in acoustics. James Candy (Univ. of California, Lawrence Livermore Natl. Lab., P.O. Box 808, L-156, Livermore, CA 94551)

Signal processing represents a technology that provides the mechanism to extract the desired information from noisy acoustical measurement data. The desired result can range from extracting a single number like sound intensity level in the case of marine mammals to the seemingly impossible task of imaging the complex bottom in a hostile ocean environment. Some of the latest approaches to solving acoustical processing problems including sophisticated Bayesian processors in architectural acoustics, iterative flaw removal processing for non-destructive evaluation, time-reversal imaging for buried objects and time-reversal receivers in communications as well as some of the exciting breakthroughs using so-called blind processing techniques for deconvolution are discussed. Processors discussed range from the simple to the sophisticated as dictated by the particular application. It is shown how processing techniques are crucial to extracting the required information for success in the underlying application. 
3pID2. Determining the sources of sounds: Psychological acoustics. William A. Yost (Parmly Hearing Inst., Loyola Univ. Chicago, 6525 N. Sheridan Rd., Chicago, IL 60626, wyost@luc.edu)

Perhaps the most important task performed by an organism's sensory system is determining objects in the world surrounding the organism. This is a challenging task for the auditory system, especially when many objects exist at the same time. The peripheral auditory system provides the neural code for the spectral-temporal structure of the complex sound impinging on the ears. It does not code for the sources of the sound. Neural centers beyond the periphery most analyze this spectral-temporal code in order to form perceptual images that make up the auditory scene. Recent psychoacoustical and perceptual research on sound source determination and segregation will be described. This research is just beginning to suggest ways in which the auditory scene is determined. [Work sponsored by NIDCD.]

2:45

3pID3. Hot topics in engineering acoustics. Stephen C. Thompson (Knowles Electron., LLC, 1151 Maplewood Dr., Itasca, IL 60143)

From the ASA Handbook, the technical committee on Engineering Acoustics is "concerned with the evolution and improvement of acoustical techniques and apparatus, and with the promotion of new applications of acoustics for useful purposes." The interest of acousticians in apparatus and techniques is at least as old as the society, as can be verified by a quick scan of the first volume of the journal. Active development continues in areas that include new materials for high power sonar devices, new structures for hearing aid transducers, the announcement of the first commercial micromachined silicon microphone, multimedia and 3-D audio applications, and more. This paper is an abbreviated report on a few of these developments.

WEDNESDAY AFTERNOON, 5 JUNE 2002

KINGS GARDEN NORTH, 1:00 TO 2:00 P.M.

\title{
Session 3pSA
}

\section{Structural Acoustics and Vibration: Holography}

\author{
Sean F. Wu, Chair \\ Department of Mechanical Engineering, Wayne State University, 505 Anthony Wayne Drive, Detroit, Michigan 48202
}

\section{Contributed Papers}

1:00

3pSA1. Spherical wave approximation to arbitrary sound fields. Nassif E. Rayess (College of Eng. and Sci., The Univ. of Detroit, Mercy, Detroit, MI 48219-0900)

The ability to expand the acoustic field radiated from arbitrarily shaped objects in the form of a series of spherical wave functions has always been a point of interest. Despite the paucity of theoretical understanding, experimental and numerical work has repeatedly shown that such expansions can lead to relatively accurate approximations of the radiated sound fields. A general understanding of the problem as well as a quantitative approximation of the resulting errors are deduced from the sound radiation model based on a volume distribution of monopole sources. The errors involved in the approximation are a direct result of the presence of monopole sources outside the largest inscribed sphere. It is shown that under many circumstances, such errors are relatively small and can be mitigated further by the proper choice of the method used to determine the coefficients of the expansion. The collocation method with an appropriate sampling scheme has been used with a great deal of success to determine the expansion coefficients in acoustic holography problems.

\section{1:15}

3pSA2. HELS based near-field acoustic holography for a highly nonspherical structure. Manmohan Moondra and Sean Wu (Dept. of Mech. Eng., Wayne State Univ., 5050 Anthony Wayne Dr., Detroit, MI 48202)

The Helmholtz Equation Least Squares (HELS) method [Wu and Yu, J. Acoust. Soc. Am. 104, 2054-2060 (1998); Wu, ibid. 107, 2511-2522 (2000)] are validated experimentally in both exterior and interior regions of a highly nonspherical structure. This is contrary to the common belief that expansion solutions based on spherical coordinates are valid within the region bounded by spheres. Detailed explanations for this phenom- enon, known as the Rayleigh hypothesis, are given in a separate paper. Shown here are the experimental validations of the acoustic fields reconstructed by the HELS method for a cabinet with overall dimensions 2.05 $\mathrm{m} \times 1.05 \mathrm{~m} \times 0.75 \mathrm{~m}$. A loudspeaker is placed inside the cabinet. The white noise produced by the speaker is measured by an array of $48 \mathrm{mi}-$ crophones stationed outside. The acoustic pressures in the near- and farfields are reconstructed and validated with respect to those measured at the same locations. Also reconstructed is the normal component of the timeaveraged acoustic intensity, from which the transmission paths are identified. Such transmission paths cannot be revealed based on the acoustic pressure distribution alone because pressure is a scalar quantity. The interior acoustic pressures are reconstructed with the speaker placed outside and microphone array inside the cabinet. [Work supported by NSF.]

\section{1:30}

3pSA3. Reconstruction of acoustic radiation from a finite object in nonfree space. Sean Wu and Xiang Zhao (Dept. of Mech. Eng., Wayne State Univ., 5050 Anthony Wayne Dr., Detroit, MI 48202)

The near-field acoustic holography (NAH) techniques developed so far have focused on reconstruction of acoustic radiation from a vibrating structure in either an unbounded exterior region or an enclosed region so that it can be solved as an interior problem. In practice, many vibrating structures are mounted on a solid foundation or in the vicinity of reflecting surfaces. For NAH to become a robust diagnostic tool, the effect of acoustic pressure reflection from nearby surfaces must be considered. In this paper, the Helmholtz equation least-squares (HELS) method [Wu, J. Acoust. Soc. Am. 107, 2511-2522 (2000)] will be modified which expresses the field acoustic pressure as a superposition of both out-going and in-coming spherical waves. The latter will be used to approximate the acoustic pressure reflection from nearby surfaces. To guarantee a convergence of the reconstructed acoustic field, a constrained minimization will be conducted with respect to the expansion functions to determine the 
optimal numbers of expansion terms for both out-going and in-coming waves. This concept will also be extended to reconstruction of acoustic radiation from an arbitrarily shaped vibrating structure for which the conventional HELS formulation has been shown to have difficulties to yield satisfactory reconstruction. [Work supported by NSF.]

\section{$1: 45$}

3pSA4. On the behavior of solutions obtained by the HELS method inside the minimum sphere. Tatiana Semenova and Sean F. Wu (Dept. of Mech. Eng., Wayne State Univ., 5050 Anthony Wayne Dr., Detroit, MI 48202)

Previous experimental and numerical studies have shown that the Helmholtz equation least-squares (HELS) method [Wu, J. Acoust. Soc. Am. 107, 2511-2522 (2000)] can be an effective methodology for nearfield acoustical holography. However, results also demonstrated that the accuracy of reconstruction on a highly nonspherical surface might be unsatisfactory. The validity of the HELS method on these highly nonspherical surfaces has become a controversial topic, just like the Rayleigh hypothesis for acoustic scattering on a corrugated surface. In this paper, the validity of solutions obtained by the HELS method will be examined for acoustic radiation from an infinite column with a rectangular cross section. The acoustic field inside the minimal circle that encircles the column will be reconstructed. The convergence of solutions both on the surface and inside the minimum circle will be checked. Moreover, a modified HELS formulation will be used, which describes the acoustic pressure as a superposition of both out-going and in-coming spherical waves. The nature of the solutions thus obtained will be examined, and the dependence of convergence of the HELS solution on the validity of the Rayleigh hypothesis and on the measurement locations will be investigated. [Work supported by NSF.]

WEDNESDAY AFTERNOON, 5 JUNE 2002

GRAND BALLROOM 1, 10:00 TO 12:00 NOON

\title{
Plenary Session, Business Meeting, Awards Ceremony
}

\author{
William M. Hartmann, President \\ Acoustical Society of America
}

\section{Business Meeting}

\author{
Presentation of Certificates to New Fellows \\ Rachel K. Clifton \\ Kerry W. Commander \\ Brian H. Houston \\ Patrick M. Hurdle \\ Darlene R. Ketten \\ Birger Kollmeier \\ Asbjörn Krokstad \\ David E. Marsh \\ Andrzej Rakowski \\ David E. Marsh \\ Andrzej Rakowski \\ W. John Richardson \\ Nicholas Rott \\ Christopher Shera \\ Leon H. Sibul \\ Andrea M. Simmons \\ David C. Swanson \\ Lynne A. Werner
}

\section{Announcement of Prize}

Bruce Cornuelle, 2002 Medwin Prize in Acoustical Oceanography

\section{Presentation of Awards}

\author{
R. Bruce Lindsay Award to James M. Finneran \\ R. Bruce Lindsay Award to Thomas J. Royston
}

Silver Medal in Psychological and Physiological Acoustics to Neal F. Viemeister

Gold Medal to Robert E. Apfel

Gold Medal to Tony F. W. Embleton 